\title{
. \\ A Systematic Review of the Safety, Feasibility and Benefits of Exercise for Patients with Advanced Cancer
}

\author{
Nico De Lazzari ${ }^{1, * \mathbb{D}}$, Timo Niels ${ }^{2}$, Mitra Tewes ${ }^{1}$ and Miriam Götte ${ }^{3}$ (D) \\ 1 Department of Medical Oncology, West German Cancer Center, University Hospital Essen, \\ 45147 Essen, Germany; Mitra.tewes@uk-essen.de \\ 2 Department I of Internal Medicine, Center of Integrated Oncology Aachen Bonn Cologne Düsseldorf, \\ University Hospital of Cologne, 50937 Cologne, Germany; timo.niels@uk-koeln.de \\ 3 Department of Pediatric Hematology/Oncology, Clinic for Pediatrics 3, Center for Child and Adolescent \\ Medicine, West German Cancer Center, University Hospital Essen, 45147 Essen, Germany; \\ Miriam.goette@uk-essen.de \\ * Correspondence: Nico.deLazzari@uk-essen.de
}

check for updates

Citation: De Lazzari, N.; Niels, T.; Tewes, M.; Götte, M. A Systematic Review of the Safety, Feasibility and Benefits of Exercise for Patients with Advanced Cancer. Cancers 2021, 13, 4478. https://doi.org/10.3390/ cancers 13174478

Academic Editor: Fabio Carraro

Received: 13 July 2021

Accepted: 1 September 2021

Published: 6 September 2021

Publisher's Note: MDPI stays neutral with regard to jurisdictional claims in published maps and institutional affiliations.

Copyright: (c) 2021 by the authors. Licensee MDPI, Basel, Switzerland. This article is an open access article distributed under the terms and conditions of the Creative Commons Attribution (CC BY) license (https:// creativecommons.org/licenses/by/ $4.0 /)$.
Simple Summary: Most advanced cancer patients suffer from severe symptoms due to cancer and medical treatment. Common symptoms are physical weakness, mental problems, and tiredness. Research has shown that exercise positively influences cancer-related side effects during and after treatment and longevity in cancer survivorship. However, exercise as a supportive therapy in advanced cancer patients is still not recommended in oncological guidelines. Therefore, the aim of this systematic review was to assess the safety, feasibility, and benefits of exercise for patients with advanced cancer. Based on the results of 14 included exercise intervention studies, we conclude that exercise is safe and feasible, seems to improve physical performance, and may lower symptoms like chronic tiredness. Early integration of exercise for advanced cancer patients should be considered as usual care as a supportive strategy.

Abstract: Exercise therapy is a common supportive strategy in curative cancer treatment with strong evidence regarding its positive effects on, for example, cancer-related fatigue, health- related quality of life, and physical function. In the field of advanced cancer patients, knowledge about exercise as a useful supportive strategy is missing. The aim of this systematic review was to evaluate the feasibility and safety of exercise interventions as well as its effects on lowering the symptom burden. We included randomized controlled trials and nonrandomized controlled trials with advanced cancer patients receiving any type of exercise intervention. After an extensive literature search (in accordance to PRIMSA guidelines) in PubMed, Cochrane Library, and SPORTDiscus, 14 studies including 940 participants with different cancer entities were eligible. The results indicated the safety of exercise. In total, 493 participants received exercise interventions, with nine adverse events and no severe adverse events. The median recruitment rate was $68.33 \%$, and adherence to exercise intervention was $86 \%$. Further research with a high-quality and larger sample size is needed to clarify the potential of exercise with advanced cancer patients. Different advanced cancer entities have distinguished symptoms, and future research should construct entities-specific trial populations to figure out the best supportive exercise interventions.

Keywords: advanced cancer; exercise; feasibility

\section{Introduction}

In Europe, 1.9 million deaths each year occur due to cancer [1]. Medical improvements achieved in the last years have led to longer survival periods in patients with incurable cancer $[2,3]$. Additional integration of palliative and supportive care results in a better quality of life and may prolong survival [3,4]. Even with limited life expectation, patients with advanced cancer can live several years. This highlights the importance of introducing 
interventions to raise patients' quality of life and lower common side effects. However, a high percentage of advanced cancer patients (ACPs) suffer from a high symptom burden associated with high prevalence of pain (64\%), fatigue (62\%), anorexia (34\%), constipation $(32 \%)$, weakness (32\%), and dyspnea (31\%) [5-9], resulting in psychological distress and lowered quality of life. Further supportive care options are required to improve the quality of life and lower symptom burden. Exercise therapy is a promising method with the potential to decrease symptoms and treatment side effects in cancer patients and survivors. Accompanying exercise interventions during cancer treatment and survivorship received much attention in the last decades, with over 700 unique exercise trials with more than 50,000 cancer patients [10]. Current evidence displays the unique role of physical activity in reduction of cancer risk, beneficial prevention of different cancer entities, and improving longevity among cancer survivors. Evidence shows that regular physical activity is beneficial for the prevention of several types of cancer and for increased survival rates in cancer patients [11]. Furthermore, exercise therapy improves cancer-related fatigue, pain, dyspnea, quality of life, and physical fitness, and prevents muscle loss during active systematic treatment [12-16]. Especially, aerobic exercise is known to be an effective tool to manage cancer-related fatigue within early stage cancer and adjuvant treatment [17], and also improves cardiorespiratory fitness [18]. Resistance exercise within cancer patients improves lower limb and upper limb strength, muscle mass, and bone density [19,20]. In addition, resistance exercise during chemotherapy leads to higher completion rates [21]. Combined aerobic and resistance exercise can improve psychosocial health-related outcomes, e.g., anxiety and depression [22]. Exercise during chemotherapy or radiotherapy is also able to reduce harmful side effects of systematic treatment with decreased expression of symptoms [23-25]. However, most research on the therapeutical effects of exercise has been conducted during survivorship or during active cancer treatment of early stage cancer disease, leading to a large research gap in ACPs undergoing palliative treatment. Exercise therapy in incurable cancer patients is not yet approached as a standard recommendation $[11,26]$. This review aims to systematically summarize the evidence of exercise interventions with ACPs to support medical professionals, physiotherapists, and exercise therapist to develop evidence-based solutions to lower the symptom burden in ACPs. The last review on this topic was published by Heywood et al. in 2018 [27]. In addition to the evidence summarized in this 2018 publication, we also searched for the most recent studies from this rapidly evolving topic with the difference that only randomized (RCTs) and nonrandomized controlled trials (CTs) with 100\% ACPs in trial were included. However, no review has analyzed different exercise modalities in exclusively ACPs with that strict inclusion criteria.

\section{Materials and Methods}

This systematic review was accomplished in accordance with the PRISMA guidelines [28], and the protocol was registered in PROSPERO database (CRD42020189850).

\subsection{Information Sources and Searches}

Sources for the conducted review were MEDLINE (PubMed), Cochrane Library, and EBSCO (SPORTDiscus). Searches were carried out on 10 July 2020. We performed an identical rerun on 16 June 2021. Main terms for the database search were as follows: ((neoplasms [MeSH] OR advanced cancer OR stage IV cancer Or incurable cancer OR metastatic cancer OR terminal cancer OR advanced tumor OR metastatic tumor OR terminal tumor OR incurable tumor OR stage IV tumor OR advanced tumour OR metastatic tumour OR terminal tumour OR incurable tumour OR stage IV tumour) AND (exercise [MeSH] OR aerobic exercise OR endurance exercise OR mobility exercise OR strength exercise OR exercise therapy OR aerobic training OR endurance training OR mobility training OR strength training OR training therapy) AND (feasibility OR fatigue OR quality of life OR strength OR endurance) AND (usual care OR palliative treatment)). Search terms were adapted to the respective databases. There were no restrictions regarding publication 
date. Written language of the publications was restricted to English or German, because of linguistic barriers of the authors.

\subsection{Study Selection}

Two independent authors (TN and NDL) screened databases for eligible articles. Studies were eligible if matching the following inclusion criteria: (a) randomized controlled trials (RCTs) or nonrandomized controlled trials (CTs); (b) human population $\geq 18$ years with diagnosed advanced cancer (Stage III or IV); (c) exercise interventions involving endurance and/or resistance exercise; (d) control group received usual/standard care (no intervention) or physiotherapy, and (e) reported at least one of the following outcomes: primary outcome: feasibility of exercise intervention in ACPs based on adherence, dropout rate, recruitment number, and adverse events; secondary outcomes: quality of life, fatigue, and physical performance assessed by objective measures, e.g., aerobic and strength tests. Combined interventions, e.g., nutrition support and exercise or psychosomatic and exercise, were excluded. Study abstracts and protocols were not eligible. After removing duplicates, two authors (TN and NDL) independently screened titles and abstracts. In case of disagreement for eligibility of titles and abstract or final inclusion, authors discussed until a consensus was reached. If no consensus could be reached, a senior author (MG) was contacted. Full text article screening of the eligible abstracts was conducted by (TN and NDL), accordingly. If full text articles did not contain sufficient data regarding relevant inclusion criteria (i.e., cancer stage), corresponding authors were contacted via email. The collecting of studies and screening were carried out with Citavi Version 6.0. (Swiss Academic Software GmbH, Zürich, Switzerland)

\subsection{Outcome Assessment}

As primary outcome, we evaluated the feasibility of exercise interventions in ACPs, which was assessed by adherence to intervention, drop-out rate, recruitment rate, and adverse events occurred during trial participation.

Secondary outcomes included questionnaire-based assessments for fatigue, quality of life, pain, sleep, and physical activity levels, and objective measurements of body composition, physical activity levels, and physical function.

\subsection{Data Extraction and Quality Assessment}

All data described in the protocol were extracted independently by two reviewers and inserted into a previously developed excel sheet. If the results were not matching, a consensus discussion between the two authors (TN and NDL) was carried out until final agreement. Extracted information included study type, study year, study population, sample size and participants' demographics (age), outcomes and times of measurement, intervention type, duration, intensity and frequency of exercise, cancer type, stage of cancer, fatigue scores, feasibility measurement in form of adherence, drop-outs, recruitment rate, adverse events, outcomes of aerobic exercise, strength exercise, mobility exercise, and functional tests. Statistical variables extracted: mean, standard deviation, and $p$-values.

To determine the risk of bias in the randomized controlled trials, two authors (TN and NDL) independently assessed risk of bias for all included studies from five domains: Bias arising from randomization process, bias due to deviations from intended interventions, bias due to missing outcome data, bias in measurement of the outcome, and bias in selection of the reported result with the Cochrane risk of bias assessment (RoB 2.0, Cochrane, London, UK) [29]. Risk of bias assessment for clinical controlled trials (CCTs) was carried out by ROBINS-I tool (Cochrane, London, UK) [30] with seven bias domains (confounding, selection of participants, classification of interventions, bias due to deviations from intended interventions, missing data, measurement of outcomes, and selection of reported results). Disagreement was solved by discussion between two authors TN and NDL. 


\subsection{Data Syntheses and Statistical Analyses}

After extracting the data of the included studies, a large heterogeneity was revealed with respect to included cancer types and measurements of the secondary effectiveness parameters due to different questionnaires or different assessments. This situation did not allow a meta-analysis of the existing studies.

\section{Results}

\subsection{Characteristics of Included Trials and Participants}

The database search resulted in $n=1327$ records. After removing duplicates, $n=959$ articles were screened for titles and abstracts, resulting in $n=186$ records assessed for eligibility. After screening the full-text articles, $n=172$ records were excluded (Figure 1: PRISMA flow diagram copyright by Cochrane group). Rerun search revealed $n=200$ more eligible studies, which were screened for titles and abstracts, with $n=6$ deemed eligible. Full-text screening exhibited no additional eligible studies. We contacted 12 authors due to insufficient information, with six responders, resulting in $n=14$ records for inclusion. In total, 12 randomized controlled trials [31-43] and two controlled trials [44,45] were included, and the results are summarized in Tables 1 and 2. In total, $n=940$ patients were included, of which $n=493$ patients received the exercise intervention and $n=447$ patients formed the control groups. According to nine studies, patients were defined as ACPs by tumor stage IV, one study defined advanced after first line of treatment with palliative intent, one after clinical assessment, e.g., computed tomography, magnetic resonance imaging, and histological examination, and one study defined advanced with a short lifetime expectancy. Tumor entities were mixed in seven studies [31-33,36,40,41,43,44], two examined prostate cancer [35,38], two lung cancer [34,42], one nasopharyngeal cancer [37], one colorectal [39] and one investigated head and neck cancer [45]. Out of the 14 studies [31-45], 9 compared an exercise intervention with usual care [31,32,35-39,41,45], out of which $n=5$ studies did not provide proper information about the actual meaning of usual care [32,37,40,42]. One study compared exercise to conventional physiotherapy [34], and one study compared exercise with usual care containing aspiration exercise and hot roll treatments [33,43]. 

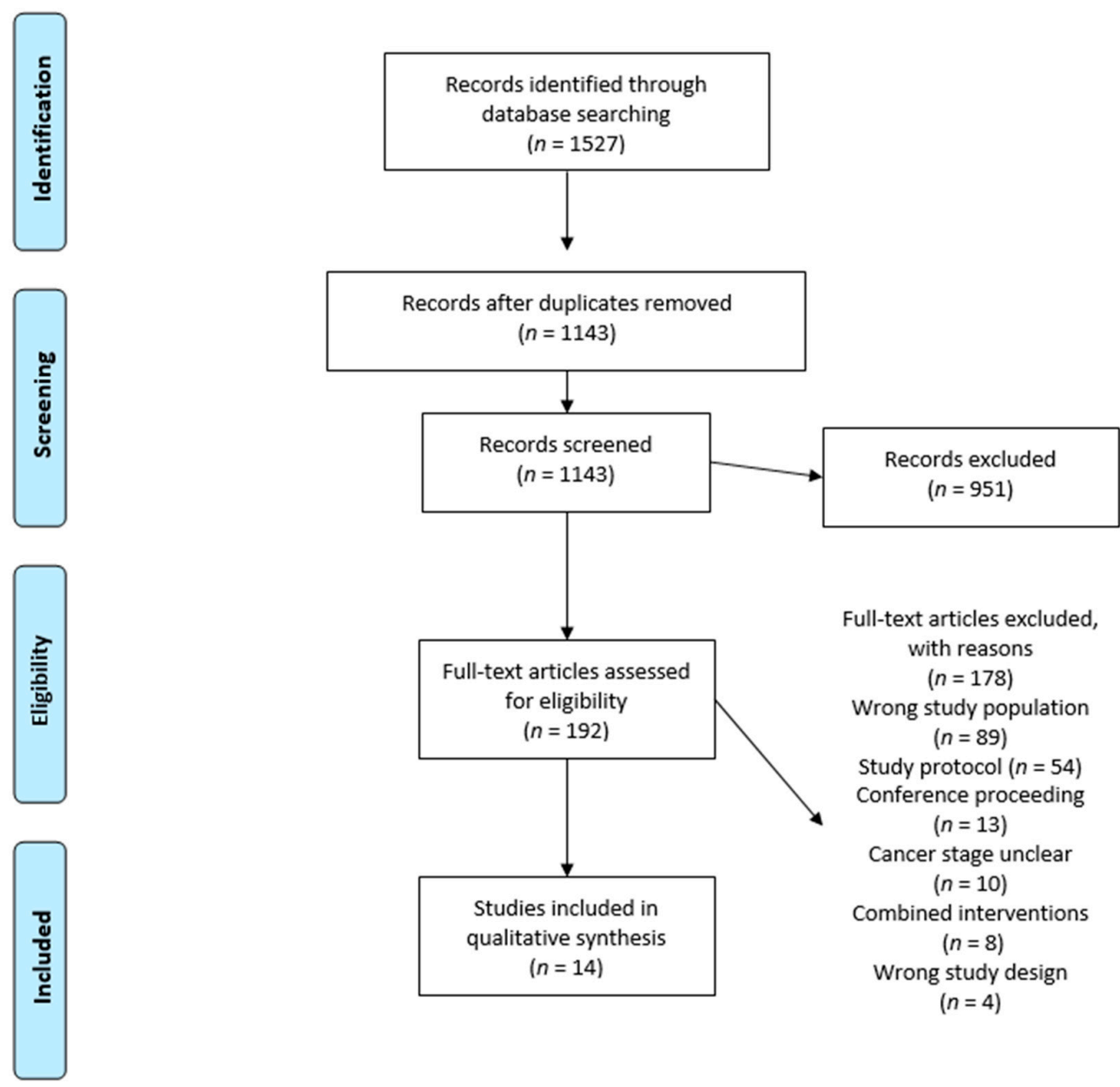

Figure 1. Preferred Reporting Items for Systematic Reviews and Meta-Analysis (PRISMA) flow diagram with number of studies identified and selected for inclusion in the systematic review [28]. Creative Commons Attribution License with permission if cited.

\subsection{Quality Assessment}

Quality assessment of the included studies is shown in Figures 2 and 3. Overall, after assessment of RoB 2.0, three studies showed an overall low risk of bias [35,38,39], nine studies showed an overall rating with some concern [31-33,35,37,40-43], and one study was rated with a high risk [34]. This rating results from concerns with the randomization process and deviation from intended interventions. Two studies were assessed using the ROBINS-I tool, with one study having an overall low risk of bias [45]. A serious risk of bias was found in the second study [44], especially bias due to confounding, bias in classification of intervention, and bias due to deviations from intended intervention.

Bias arising from the randomization process Bias due to deviations from intended interventions Bias due to missing outcome data Bias in measurement of the outcome Bias in selection of the reported result

Overall risk of bias

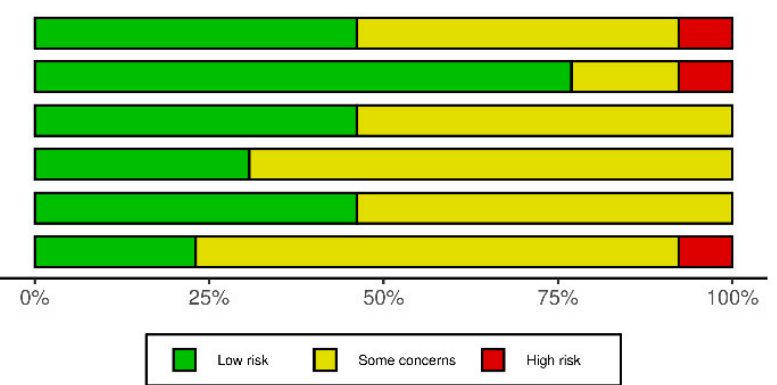

Figure 2. Cont. 


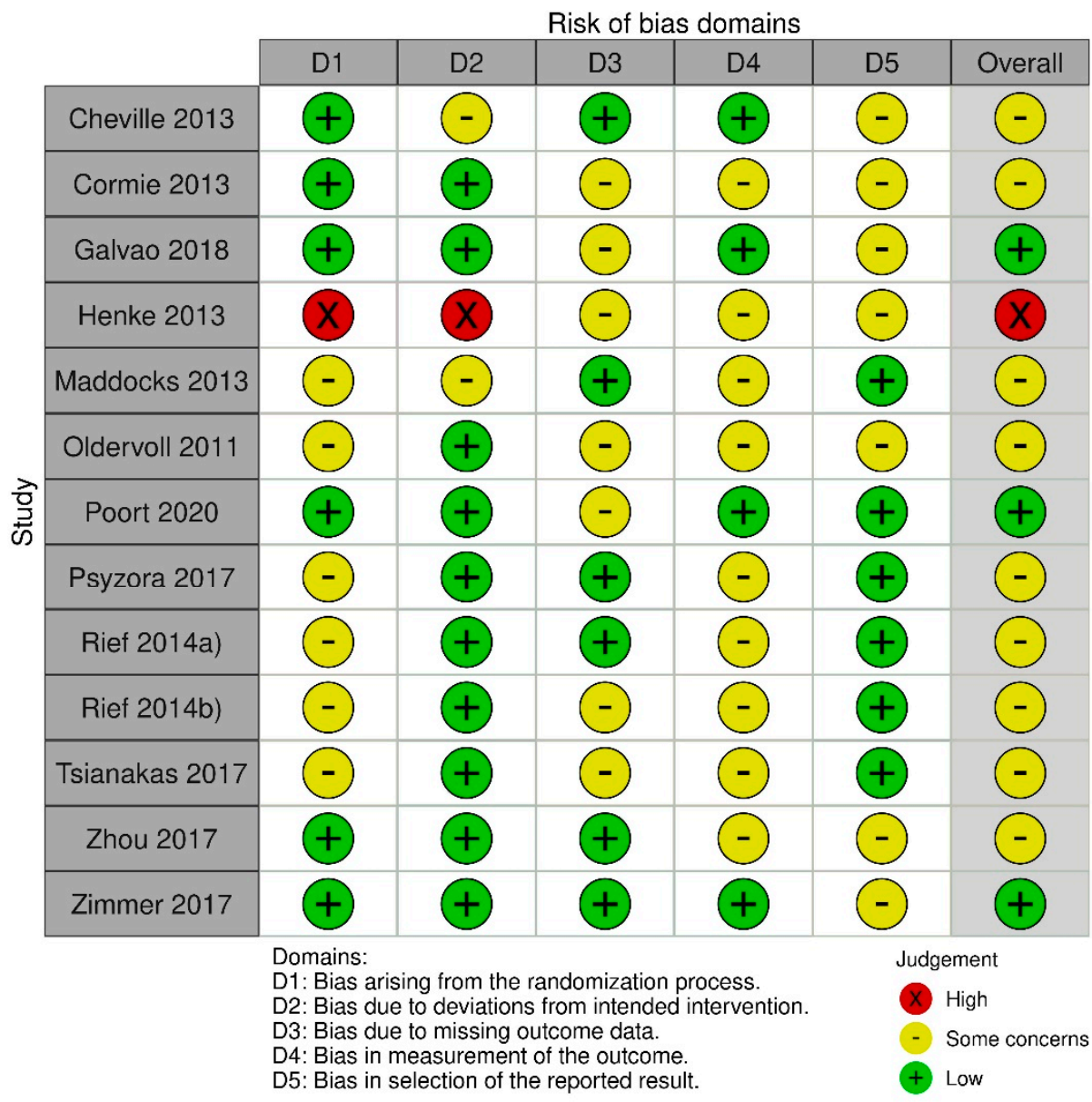

Figure 2. Risk of bias for randomized controlled trials (RoB 2.0).

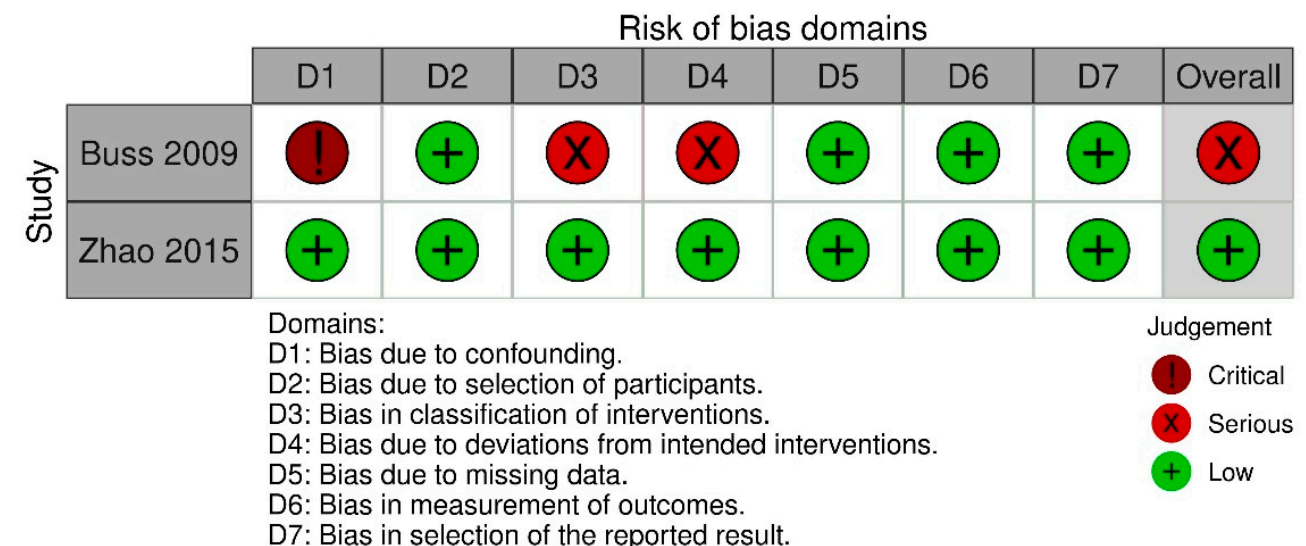

Figure 3. Cont. 


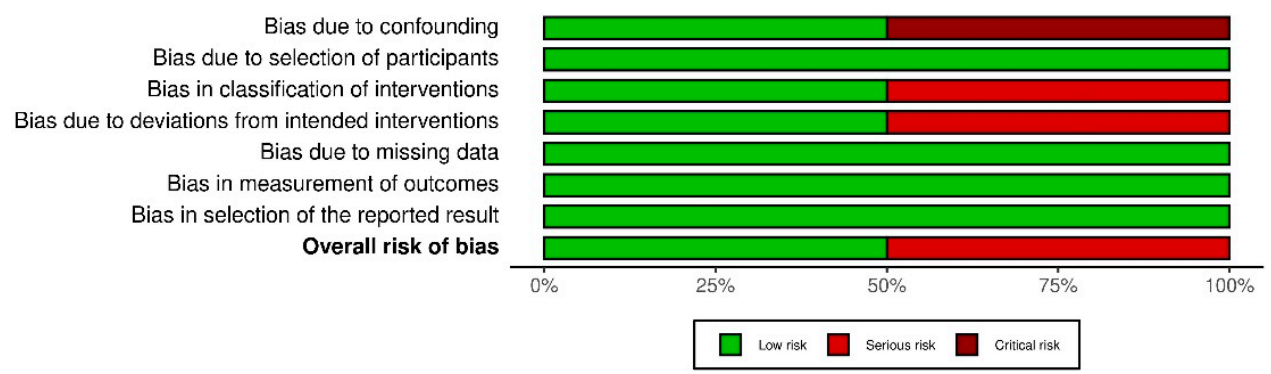

Figure 3. Risk of bias assessment for nonrandomized trials (ROBINS-I tool).

\subsection{Intervention Description}

Interventions in the observed studies mostly consisted of a combination of aerobic and strength exercise [31-33,36,38,39,45]. Two studies focused only on strength exercise [35,44], while two studies carried out aerobic walking exercise [40,41], one absolved Thai Chi exercise [37], one fulfilled kinesiotherapy exercise [44], and one focused on neuromuscular electrical stimulation (NMES) of the quadriceps [42]. Duration of intervention ranged from 2 weeks to 14 weeks. The intervention lasted for 12 weeks in four studies [35,36,38,40], followed by eight weeks in three studies [31,32,39], two studies completed a two-week training intervention $[33,41,43]$, and one study duration was between 8 and 11 weeks (3-4 chemotherapy cycles). The longest study duration was 14 weeks [45], while one study exercised for 4 weeks [44] and one did not give proper information on the duration of the intervention [37]. Intensity of the exercise interventions was described precisely in three studies $[34,36,39]$. Strength exercise was applied from $50 \%$ to $80 \%$ of the maximal capacity (one-repetition maximum) with progressive increase. The intensity of aerobic exercise was precisely described in the same three studies [34,36,39]. Other studies [31-33,35,37,38,40-45] did not describe the intensity of the fulfilled interventions accurately. Exercise sessions per week varied between two times per week to seven times per week with different amounts of workload (active exercise minutes/week). Of the $n=14$ [31-45] different interventions, $n=11$ [32,34-39,41] were supervised exercise interventions. In one study, home-based training was performed [31]. Tsianakas et al. [40] conducted a group intervention supervised by volunteers. The participants of Maddocks et al. [42] completed the intervention in their own administration. Zhao et al. [45] and Rief et al. $[33,43]$ completed a period of supervised training followed by home-based training.

\subsection{Primary Outcome: Feasibility and Safety}

The primary aim of the systematic review was to evaluate if structured exercise is feasible and safe in ACPs. Adverse events were defined as any injury that occurred related to exercise. Withdrawal from trial through disease-related reasons, for example, death due to cancer, was not considered as an adverse event related to exercise.

Recruitment rates were described in twelve studies [31-38,40,42-45], while two did not mention the screening and recruitment rates [39,41]. Overall, recruitment revealed a median of $68.3 \%$ (range: $25-87.6 \%$ ). Four studies demonstrated a recruitment rate around $50 \%$ or lower $[32,36,40,42]$. Studies with a high recruitment rate $(>60 \%)$ pointed out a low number of patients screened in the study conducted [31,33-35,43,45]. Barriers to recruitment were described in 12 studies [31-38,40,42-45]. In total, 544 declined trial participation. The most frequent reason for a nonparticipation was patients refused or declined $(n=269)$ participation. This was followed by health-related deterioration or conditions that were excluded because of the trial protocol $(n=82)$. Another reason for nonparticipation was the distance to study site. Overall, $n=45$ patients declined because of travel distance, followed by $n=36$ patients had no time for trial participation or were already actively involved in exercise $(n=14)$. However, there was also a big group of ACPs where the reasons were unknown $(n=76)$. 
Table 1. Study characteristics of randomized controlled trials.

\begin{tabular}{|c|c|c|c|c|c|c|c|c|c|c|c|c|c|}
\hline Author & Cancer Entities & Age & Treat & $\begin{array}{c}n \\
(\mathrm{EX} / \mathrm{C})\end{array}$ & $\begin{array}{l}\text { Description of } \\
\text { Intervention }\end{array}$ & $\begin{array}{c}\text { Exercise } \\
\text { Modalities }\end{array}$ & Control & $\begin{array}{l}\text { Recruit-ment } \\
\text { (Participants/ } \\
\text { Eligible) }\end{array}$ & $\begin{array}{l}\text { Drop-Out } \\
\text { Rate: }\end{array}$ & Adherence & $\mathrm{AE}$ & $\begin{array}{c}\text { EX vs. UC } \\
\uparrow=\text { Improvement } \\
\downarrow=\text { Worsening }\end{array}$ & $\begin{array}{l}\text { EX vs. UC } \\
\text { (Non Sign.) }\end{array}$ \\
\hline $\begin{array}{l}\text { Cheville et al., } \\
\text { 2013 } \\
\text { [31] }\end{array}$ & $\begin{array}{c}\text { Lung and } \\
\text { colorectal } \\
\text { cancer; Stage IV } \\
(n=32 \text { colon } \\
\text { cancer; } n=34 \\
\text { lung cancer })\end{array}$ & $\begin{array}{c}\text { Ex: } 63.8 \pm \\
12.5 ; \\
\text { C: } 65.5 \pm 8.9\end{array}$ & $\begin{array}{l}\text { CTx } \\
\text { RTx } \\
\text { MAB }\end{array}$ & $\begin{array}{c}66 \\
(33 / 33)\end{array}$ & $\begin{array}{c}\text { 8-wk } \\
\text { homebased REX } \\
\text { and walking } \\
\text { exercise }\end{array}$ & $\begin{array}{c}\text { 4x/wk: } \\
\text { incremental } \\
\text { Walking: } 20 \mathrm{~min}, \\
\text { briskly, } 3.5 \mathrm{mets}, \\
\text { REX: upper/lower } \\
\text { body split, } \\
5 \text { exercise; } \\
10-15 \text { reps }\end{array}$ & Usual care & $\begin{array}{l}\text { 66/93: } \\
(70.9 \%)\end{array}$ & $\begin{array}{c}\text { EX: } 7 / 33 \\
(21 \%) \\
C: 3 / 33 \\
(9.9 \%), \\
\text { in total } 10 / 66 \\
(15.1 \%)\end{array}$ & $\begin{array}{l}20 / 26(76.9 \%) \\
\text { pts reached } \\
\text { requirement } \\
\quad \text { for } \\
\text { participation }\end{array}$ & 0 & $\begin{array}{c}\uparrow \text { AM-PAC } \\
\text { mobility }(p=0.02) \\
\uparrow \text { Fatigue (FACT-F) } \\
(p=0.03) \\
\uparrow \text { Sleep (NRS) } \\
(p=0.05)\end{array}$ & $\begin{array}{c}\square \text { Ambulatory } \\
\text { Post Acute } \\
\text { Care Daily } \\
\text { Activities Short } \\
\text { Form (AM-PAC } \\
\text { CAT) } \\
\square \text { QoL (FACT-G) }\end{array}$ \\
\hline $\begin{array}{l}\text { Cormie et al., } \\
2013 \infty \\
{[35]}\end{array}$ & $\begin{array}{l}\text { Prostate cancer; } \\
\text { secondary bone } \\
\text { metastases } \\
(n=20)\end{array}$ & $\begin{array}{l}\text { Ex: } 73.1 \pm 7.5 ; \\
\text { C: } 71.2 \pm 6.9\end{array}$ & $\begin{array}{l}\text { Prev. } \\
\text { AST } \\
\text { RTx } \\
\text { SX }\end{array}$ & $\begin{array}{c}20 \\
(10 / 10)\end{array}$ & $\begin{array}{c}\text { 12-wk } \\
\text { suplow-level } \\
\text { AEX and REX } \\
\text { targeting major } \\
\text { muscle groups }\end{array}$ & $\begin{array}{l}\text { 2x/wk; REX on } \\
\text { machines; } \\
\text { 8-12 reps, } 2-4 \text { sets, } \\
\sim 60 \text { min, } \\
\text { Low-level aerobix } \\
\text { exercise and } \\
\text { stretching }\end{array}$ & Usual care & 20/27: (74\%) & $\begin{array}{c}\text { EX: } 2 / 10 \\
(20 \%) \\
\text { C: } 3 / 10 \\
(30 \%) \\
\text { in total } 5 / 20 \\
(25 \%)\end{array}$ & $\begin{array}{c}83 \% \\
\text { attendance } \\
\text { (mean: } 20.2 \pm \\
7.6 \text { out of } \\
24 \text { sessions); } \\
70 \% \text { pts } \\
\text { completing } \\
24 / 24 \text { ses- } \\
\text { sions }\end{array}$ & 0 & $\begin{array}{c}\uparrow \text { Physical } \\
\text { function } \\
(p=0.016) \\
\uparrow 400-\mathrm{m} \text { Walk } \\
(p=0.010) \\
\uparrow 6 \mathrm{MWT} \text {; usual } \\
\text { pace }(p=0.001) \\
\uparrow \text { Body lean mass } \\
(p=0.026) \\
\uparrow \text { Lean mass } \\
(p=0.003)\end{array}$ & $\begin{array}{c}\boxminus \text { Fatigue } \\
\square \text { QoL }\end{array}$ \\
\hline $\begin{array}{l}\text { Galvao et al., } \\
\quad 2018 \\
{[38]}\end{array}$ & $\begin{array}{l}\text { Prostate cancer; } \\
\text { bone metastases } \\
\quad(n=57)\end{array}$ & $\begin{array}{l}\text { Ex: } 69.7 \pm 7.6 ; \\
\text { C: } 70.4 \pm 9.3\end{array}$ & $\begin{array}{l}\text { AST } \\
\text { CTx }\end{array}$ & $\begin{array}{c}57 \\
(28 / 29)\end{array}$ & $\begin{array}{l}\text { 12-wk sup, } \\
\text { combined AEX, } \\
\text { REX, and } \\
\text { flexibility } \\
\text { exercise }\end{array}$ & $\begin{array}{c}\text { 3x/wk: } \\
\text { REX: } \\
\text { progressively; } \\
\text { 10-12 reps; } 3 \text { sets } \\
\text { AEX: } 20-30 \text { min; } \\
\text { Cycling/treadmill; } \\
\text { 60-85\% of max. } \\
\text { HR, Flexibility EX: } \\
\text { static stretching; } \\
\text { 2-4 reps, each } \\
\text { muscle group } \\
\text { 30-60 s }\end{array}$ & Usual care & $\begin{array}{l}\text { 57/103: } \\
(55.3 \%)\end{array}$ & $\begin{array}{c}\text { EX: } 5 / 28 \\
(17.8) \\
C: 3 / 29 \\
(10.3 \%) \\
\text { in total } 8 / 57 \\
(14 \%)\end{array}$ & $\begin{array}{c}\text { Session: } \\
32 \pm 10 / 36 \\
89 \% \\
\text { attendance }\end{array}$ & 0 & $\begin{array}{c}\uparrow p \text { physical } \\
\text { function (NBS) } \\
(p=0.028) \\
\uparrow \text { leg extension } \\
(p=0.033)\end{array}$ & $\begin{array}{c}\square 6 \text { MWT } \\
\square 400 \text { m walk test } \\
\square \text { Up and Go Test } \\
\square \text { SOT } \\
\square \text { Lean mass } \\
\square \text { Body fat mass. } \\
\square \text { Fatigue } \\
\text { (FACT-F) }\end{array}$ \\
\hline $\begin{array}{l}\text { Henke et al., } \\
2013 \\
{[34]}\end{array}$ & $\begin{array}{c}\text { Lung cancer } \\
\text { Stage } \\
\text { IIIA/IIIB/IV } \\
(n=46)\end{array}$ & n.a. ${ }^{*}$ & CTx & $\begin{array}{c}46 \\
(25 / 21)\end{array}$ & $\begin{array}{l}3 \text { chemotherapy } \\
\text { cycles long: Sup } \\
\text { Combined REX } \\
\text { and AEX }\end{array}$ & $\begin{array}{c}7 x / \text { wk } \\
\text { REX: resistance } \\
\text { bands; } 50 \% \text { of } \\
\text { max. capacity. } \\
10 \text { reps, } 3 \text { sets } \\
5 \times / \text { wk: } \\
\text { AEX: } 6 \text { min } \\
\text { walking moderate } \\
\text { intensity (55-70\% } \\
\text { of HRmax) }\end{array}$ & $\begin{array}{l}\text { Conventional } \\
\text { physiother- } \\
\text { apy }\end{array}$ & $\begin{array}{l}46 / 70: \\
(65.7 \%)\end{array}$ & $\begin{array}{c}\text { EX: } 6 / 24 \\
(25 \%) \\
\text { C: } 9 / 20(45 \%) \\
\text { in total 15/44 } \\
(34 \%) \\
n=2 \\
\text { dropouts } \\
\text { before ran- } \\
\text { domization }\end{array}$ & n.a. * & n.a. * & $\begin{array}{c}\uparrow 6 \text { MWT }(p<0.05) \\
\uparrow \text { Staircase } \\
\text { walking }(p=0.05) \\
\uparrow \text { Physical } \\
\text { functioning } \\
\text { (C-30/LC-13, } \\
p=0.025) \\
\uparrow \text { Cognitive } \\
\text { functioning } \\
\text { (C-30/LC-13, } \\
p=0.050\end{array}$ & $\begin{array}{c}\square \text { Global health } \\
\text { status/QoL } \\
\square \text { Role } \\
\text { functioning (C-30) } \\
\square \text { Emotional } \\
\text { functioning (C-30) } \\
\square \text { Symptom } \\
\text { Scales (C-30/L-13) }\end{array}$ \\
\hline
\end{tabular}


Table 1. Cont

\begin{tabular}{|c|c|c|c|c|c|c|c|c|c|c|c|c|c|}
\hline Author & Cancer Entities & Age & Treat & $\begin{array}{c}n \\
\text { (EX/C) }\end{array}$ & $\begin{array}{l}\text { Description of } \\
\text { Intervention }\end{array}$ & $\begin{array}{c}\text { Exercise } \\
\text { Modalities }\end{array}$ & Control & $\begin{array}{c}\text { Recruit-ment } \\
\text { (Participants/ } \\
\text { Eligible) }\end{array}$ & $\begin{array}{l}\text { Drop-Out } \\
\text { Rate: }\end{array}$ & Adherence & $\mathrm{AE}$ & $\begin{array}{c}\text { EX vs. UC } \\
\uparrow=\text { Improvement } \\
\downarrow=\text { Worsening }\end{array}$ & $\begin{array}{c}\text { EX vs. UC } \\
\text { (Non Sign.) }\end{array}$ \\
\hline $\begin{array}{l}\text { Maddocks } \\
\text { et al., } \\
2013 \\
{[42]}\end{array}$ & $\begin{array}{l}\text { NSCLC cancer; } \\
\text { Stage IV }(n=49)\end{array}$ & $\begin{array}{c}\text { Ex: 70; } \\
\text { C: } 68 \text {, n.a. * } \\
\text { STD }\end{array}$ & CTx & $\begin{array}{c}49 \\
(30 / 19)\end{array}$ & $\begin{array}{c}3-4 \\
\text { chemotherapy } \\
\text { cycles long } \\
\text { (8-11 wk) } \\
\text { self- } \\
\text { administered } \\
\text { neuromuscular } \\
\text { electrical } \\
\text { stimulation } \\
\text { (NMES) of the } \\
\text { quadriceps } \\
\text { Muscle }\end{array}$ & $\begin{array}{c}3-7 x / \text { wk; } \\
30 \mathrm{~min} / \mathrm{session} \\
\text { each leg; } \\
\text { intensity: } 50 \mathrm{~Hz} \\
\text { frequency: } \\
350 \text { microseconds } \\
\text { pulse width; duty } \\
\text { cycle: } 11-25 \% ; \\
0-120 \mathrm{~m} \text { ) }\end{array}$ & n.a. * & $\begin{array}{c}49 / 190: \\
(25 \%)\end{array}$ & $\begin{array}{c}\text { EX: } 15 / 30 \\
(50 \%) \\
C: 6 / 19 \\
(31.5 \%) \\
\text { in total } 21 / 49 \\
(42.8 \%)\end{array}$ & $\begin{array}{c}9 / 15 \\
\text { completing at } \\
\text { least } 3 x / w k\end{array}$ & $\begin{array}{l}n=3 \\
\text { NMES- } \\
\text { related } \\
\text { muscle } \\
\text { discomfort } \\
\text { resulting in } \\
\text { dropout } \\
\text { (CTC } \\
\text { grade I) }\end{array}$ & $\begin{array}{l}\uparrow \text { Mental Fatigue } \\
\quad(p=0.03)\end{array}$ & $\begin{array}{c}\square \text { Quadriceps } \\
\text { muscle strength } \\
\square \text { Thigh lean mass } \\
\square \text { Whole Body } \\
\text { lean mass } \\
\square \text { Step count } \\
\square \text { Fatigue } \\
\square \text { QoL }\end{array}$ \\
\hline $\begin{array}{c}\text { Oldervoll } \\
\text { et al., } \\
2011 \\
{[32]}\end{array}$ & $\begin{array}{c}\text { Incurable, } \\
\text { metastatic } \\
\text { cancer, and life } \\
\text { expectancy of } \\
3-24 \text { months } \\
(n=41 \\
\text { gastrointestinal, } \\
n=23 \text { breast, } \\
n=21 \text { lung, } \\
n=16 \\
\text { urological, } n=8 \\
\text { gynaecological, } \\
n=2 \\
\text { hematological, } \\
\text { and } n=10 \text { other } \\
\text { cancer) }\end{array}$ & $\begin{array}{c}\text { Ex: } 62.6 \pm \\
11.3 ; \mathrm{C}: 62.2 \\
\quad \pm 10.7\end{array}$ & $\begin{array}{l}\text { CTx } \\
\text { RTx } \\
\text { HT } \\
\text { TT }\end{array}$ & $\begin{array}{c}231 \\
(121 / 110)\end{array}$ & $\begin{array}{l}\text { 8-wk sup, } \\
\text { combined } \\
\text { exercise }\end{array}$ & $\begin{array}{l}\text { 2x/wk; } 50-60 \text { min, } \\
\text { AEX: Warmup } \\
\text { 10-15 min + } \\
\text { Circuit EX: } \\
\text { 6-station circuit; } \\
2 \text { min on, 1min off, } \\
\text { combining } \\
\text { strength, aerobic, } \\
\text { and coordination } \\
\text { elements. }\end{array}$ & n.a. ${ }^{*}$ & $\begin{array}{l}231 / 400: \\
(57.7 \%)\end{array}$ & $\begin{array}{c}\text { EX: } 43 / 121 \\
(35.5 \%) \\
\text { C: } 25 / 110 \\
(22.7 \%) \\
\text { in total } \\
68 / 231 \\
(29.4 \%)\end{array}$ & $\begin{array}{c}69 \% \text { finished } \\
\text { pre and post } \\
\text { assessment } \\
\text { sessions: } \\
11 / 16\end{array}$ & 0 & $\begin{array}{c}\uparrow \text { Shuttle walk test } \\
(p=0.008) \\
\uparrow \text { Handgrip } \\
\text { strength }(p=0.01)\end{array}$ & $\begin{array}{c}\square \text { Total fatigue } \\
\square \text { Physical fatigue } \\
\square \text { Mental fatigue } \\
\square \text { Sit-to-stand }\end{array}$ \\
\hline $\begin{array}{c}\text { Poort et al., } \\
2020 \\
{[36]}\end{array}$ & $\begin{array}{c}\text { Cancer patients } \\
\text { with palliative } \\
\text { systemic } \\
\text { treatment } \\
(n=35 \text { breast, } \\
n=21 \\
\text { colorectal, } \\
n=19 \text { prostate, } \\
n=4 \text { renal cell, } \\
n=4 \text { ovarian, } \\
n=4 \text { melanoma, } \\
n=1 \text { bladder } \\
\text { cancer })\end{array}$ & $\begin{array}{l}\text { Ex: } 60,67 \pm \\
10.7 ; C: 63.9 \\
\quad \pm 8.9\end{array}$ & $\begin{array}{c}\text { CTx } \\
\text { HT } \\
\text { TT } \\
\text { CTx + } \\
\text { TT } \\
\text { HT + } \\
\text { TT } \\
\text { IT }\end{array}$ & $\begin{array}{c}134 \\
(42 / 46 \\
(+46 \\
\text { in } \\
\text { ARM3) }\end{array}$ & $\begin{array}{c}12-w k \\
\text { supcombined } \\
\text { REX and AEX }\end{array}$ & $\begin{array}{c}2 \mathrm{x} / \mathrm{wk}, \\
120 \mathrm{~min} / \mathrm{session}, \\
\text { AEX: } 35 \mathrm{~min}, \\
\text { Interval cycling: } \\
4 \mathrm{~min}, 60-80 \% \\
\text { HRmax } / 3 \text { min on } \\
35 \% \text { HRmax, } \\
\text { REX (large muscle } \\
\text { groups): } 35 \text { min, } \\
\text { circuit exercise; } \\
60-80 \% 1 \mathrm{RM} ; \\
8-12 \text { reps, } 3 \text { sets }\end{array}$ & Usual care & $\begin{array}{l}\text { 134/232: } \\
(57.7 \%)\end{array}$ & $\begin{array}{c}\text { EX: } 7 / 34 \\
(20.6 \%) \\
\text { C: } 3 / 46 \\
(4.7 \%) \\
\text { in total } \\
10 / 80 \\
(12.5 \%) \\
n=8 \text { did not } \\
\text { receive EX }\end{array}$ & $\begin{array}{c}\text { sessions } \\
\text { average: } \\
8 \pm 3.5 / 24 \\
\text { Treatment } \\
\text { integrity } 86 \%\end{array}$ & $\begin{array}{c}\text { EX: muscle } \\
\text { pain } \\
\text { overuse } \\
(n=4), \\
\text { cardiac } \\
\text { complaints } \\
(n=1), \\
\text { increased } \\
\text { fatigue } \\
\text { after } \\
\text { training } \\
(n=1)\end{array}$ & none & $\begin{array}{l}\square \text { Fatigue (CIS } \\
\text { and C30) } \\
\square \text { QoL (C30) } \\
\square \text { Physical } \\
\text { function (C30) } \\
\square \text { Emotional } \\
\text { function (C30) } \\
\boxminus \text { Functional } \\
\text { impairments } \\
\text { (C30) }\end{array}$ \\
\hline
\end{tabular}


Table 1. Cont

\begin{tabular}{|c|c|c|c|c|c|c|c|c|c|c|c|c|c|}
\hline Author & Cancer Entities & Age & Treat & $\begin{array}{c}n \\
\text { (EX/C) }\end{array}$ & $\begin{array}{l}\text { Description of } \\
\text { Intervention }\end{array}$ & $\begin{array}{c}\text { Exercise } \\
\text { Modalities }\end{array}$ & Control & $\begin{array}{c}\text { Recruit-ment } \\
\text { (Participants/ } \\
\text { Eligible) }\end{array}$ & $\begin{array}{l}\text { Drop-Out } \\
\text { Rate: }\end{array}$ & Adherence & $\mathrm{AE}$ & $\begin{array}{c}\text { EX vs. UC } \\
\uparrow=\text { Improvement } \\
\downarrow=\text { Worsening }\end{array}$ & $\begin{array}{c}\text { EX vs. UC } \\
\text { (Non Sign.) }\end{array}$ \\
\hline $\begin{array}{l}\text { Psyzora et al., } \\
2017 \\
{[41]}\end{array}$ & $\begin{array}{c}\text { Advanced } \\
\text { cancer patients, } \\
\text { admitted to } \\
\text { palliative care } \\
(n=15) \\
\text { Alimentary } \\
\text { system, } n=13 \\
\text { urogential } \\
\text { system, } n=8 \\
\text { mammary } \\
\text { gland, } n=6 \\
\text { Hematologica, } \\
n=6 \text { indefinite } \\
\text { origin, } n=5 \\
\text { lung, } n=5 \\
\text { central nervous } \\
\text { system, } n=1 \\
\text { mouth, } n=1 \\
\text { skin) }\end{array}$ & $\begin{array}{l}\text { Ex: } 72.4 \pm 9.5 ; \\
\text { C: } 69.3 \pm 13.7\end{array}$ & n.a. & $\begin{array}{c}60 \\
(30 / 30)\end{array}$ & $\begin{array}{l}\text { 2-wk physio- } \\
\text { therapeutic } \\
\text { exercise, MFR, } \\
\text { and PNF }\end{array}$ & $\begin{array}{l}3 \mathrm{x} / \mathrm{wk}, 30 \mathrm{~min} \text {, } \\
\text { active exercise of } \\
\text { upper and lower } \\
\text { limbs, selected } \\
\text { techniques of } \\
\text { MFR and PNF }\end{array}$ & Usual care & n.a. * & $\begin{array}{c}\text { EX: } 1 / 30 \\
(3.3 \%) \\
\text { C: } 1 / 30 \\
(3.3 \%) \\
\text { in total } 2 / 60 \\
(3.3 \%)\end{array}$ & n.a. * & n.a. ${ }^{*}$ & $\begin{array}{c}\uparrow \text { Fatigue Severity } \\
\text { (BFI and ESAS) } \\
(p<0.01)\end{array}$ & $\begin{array}{c}\square \text { Depression } \\
\quad \text { (ESAS) } \\
\square \text { Anxiety (ESAS) }\end{array}$ \\
\hline $\begin{array}{c}\text { Rief et al., } \\
2014+ \\
{[33,43]}\end{array}$ & $\begin{array}{c}\text { Cancer patients } \\
\text { with metastatic } \\
\text { progress in tho- } \\
\text { racic/lumbar } \\
\text { spine or in } \\
\text { sacrum } \\
(n=20 \text { lung, } \\
n=14 \text { prostate, } \\
n=11 \text { breast, } \\
n=3 \text { renal, } \\
n=2 \text { melanoma, } \\
n=10 \text { other })\end{array}$ & $\begin{array}{l}\text { Ex: } 61.3 \pm \\
\text { 10.1; C: } 64.1 \\
\quad \pm 10.9\end{array}$ & RTx & $\begin{array}{c}60 \\
(30 / 30)\end{array}$ & $\begin{array}{l}\text { 2-wk sup } \\
\text { isometric REX } \\
\text { followed by } \\
12 \text {-wk } \\
\text { home-based } \\
\text { training }\end{array}$ & $\begin{array}{c}5 \mathrm{x} / \mathrm{wk}, 30 \mathrm{~min} \\
\text { isometric spinal } \\
\text { training of the } \\
\text { autochthonous } \\
\text { muscles }+ \\
\text { unsupervised } 3 \mathrm{x} \\
\text { weekly home } \\
\text { training }\end{array}$ & $\begin{array}{l}\text { Usual care, } \\
\text { aspiration } \\
\text { exercise, } \\
\text { hot roll } \\
\text { treatments }\end{array}$ & 60/80: (75\%) & $\begin{array}{c}\text { EX: } 5 / 30 \\
(16.6 \%) \\
\text { C: } 7 / 30 \\
(23.3 \%) \\
\text { in total } 12 / 60 \\
(20 \%)\end{array}$ & $\begin{array}{c}\text { patients } \\
\text { completed } \\
\text { the exercise } \\
\text { protocol: } \\
25 / 30(83.3 \%)\end{array}$ & n.a. ${ }^{*}$ & $\begin{array}{c}\uparrow 30 \text { s sit-to-stand } \\
(p<0.001), \\
\uparrow \text { QoL (BM22) } \\
(p=0.01) \\
\uparrow \text { Fatigue (BM22) } \\
(p=0.01) \\
\uparrow \text { Pain (VAS) } \\
(p=0.003)\end{array}$ & $\begin{array}{c}\square \text { Functional } \\
\text { interference } \\
\text { (BM22) } \\
\square \text { Emotional } \\
\text { interference } \\
\text { (BM22) } \\
\square \text { Cognitive } \\
\text { interference } \\
\text { (BM22) } \\
\square \text { Overall survival } \\
\square \\
\text { Progression-free } \\
\text { survival }\end{array}$ \\
\hline $\begin{array}{c}\text { Tsianakas } \\
\text { et al., } \\
2017 \\
{[40]}\end{array}$ & $\begin{array}{c}\text { Recurrent } \\
\text { advancing or } \\
\text { metastatic } \\
\text { cancer: } \\
n=15 \text { prostate, } \\
n=9 \\
\text { gynecological, } \\
n=9 \\
\text { hematological, } \\
n=7 \text { breast, } \\
n=5 \text { colorectal, } \\
n=1 \text { upper } \\
\text { gastrointestinal }\end{array}$ & $\begin{array}{l}\text { Ex: } 65 \pm 11.7 \\
\text { C: } 66.2 \pm 10.2\end{array}$ & n.a. & $\begin{array}{c}42 \\
(21 / 21)\end{array}$ & $\begin{array}{l}\text { 12-wk walking } \\
\text { intervention }\end{array}$ & $\begin{array}{l}30 \text { min walking on } \\
\text { alternate days }\end{array}$ & n.a. * & $\begin{array}{l}42 / 110: \\
(38.1 \%)\end{array}$ & $\begin{array}{c}\text { EX: } 8 / 21 \\
(38.1 \%) \\
\text { C: } 7 / 21 \\
(33.3 \%) \\
\text { in total } 15 / 42 \\
(35.7 \%)\end{array}$ & n.a. * & 0 & none & $\begin{array}{l}\square \text { QoL (FACT-G) } \\
\square \text { Global fatigue } \\
\text { score } \\
(\mathrm{BFI})\end{array}$ \\
\hline
\end{tabular}


Table 1. Cont.

\begin{tabular}{|c|c|c|c|c|c|c|c|c|c|c|c|c|c|}
\hline Author & Cancer Entities & Age & Treat & $\begin{array}{c}n \\
\text { (EX/C) }\end{array}$ & $\begin{array}{l}\text { Description of } \\
\text { Intervention }\end{array}$ & $\begin{array}{c}\text { Exercise } \\
\text { Modalities }\end{array}$ & Control & $\begin{array}{c}\text { Recruit-ment } \\
\text { (Participants/ } \\
\text { Eligible) }\end{array}$ & $\begin{array}{l}\text { Drop-Out } \\
\text { Rate: }\end{array}$ & Adherence & $\mathrm{AE}$ & $\begin{array}{c}\text { EX vs. UC } \\
\uparrow=\text { Improvement } \\
\downarrow=\text { Worsening }\end{array}$ & $\begin{array}{c}\text { EX vs. UC } \\
\text { (Non Sign.) }\end{array}$ \\
\hline $\begin{array}{c}\text { Zhou et al., } \\
\text { 2017 } \\
{[37]}\end{array}$ & $\begin{array}{c}\text { Advanced } \\
\text { nasopharyngeal } \\
\text { cancer stage } \\
\text { III/IV }\end{array}$ & n.a. * & $\begin{array}{l}\text { CTx } \\
\text { RTx }\end{array}$ & $\begin{array}{c}114 \\
(57 / 57)\end{array}$ & $\begin{array}{c}\text { Tai Chi exercise } \\
\text { (24-form Yang } \\
\text { style) } \\
\text { n.a. duration }\end{array}$ & $\begin{array}{l}5 \mathrm{x} / \mathrm{wk} 60 \mathrm{~min} \\
(10 \mathrm{~min} . \text { warm up, } \\
30 \mathrm{~min} \text { Tai Chi } \\
\text { exercise, } 10 \mathrm{~min} \\
\text { breath and } \\
\text { mediation; } 10 \mathrm{~min} \\
\text { relaxation })\end{array}$ & Usual care & $\begin{array}{l}\text { 114/130: } \\
\text { (87.6\%) }\end{array}$ & $\begin{array}{c}\text { EX: } 15 / 57 \\
(26.3 \%) \\
\text { C: } 16 / 57 \\
(28 \%) \\
\text { in total } \\
31 / 114 \\
(27.1 \%)\end{array}$ & n.a. * & 0 & $\begin{array}{c}\uparrow \text { Fatigue } \\
\text { (MFSI-SF) } \\
\text { ( } p<0.05) \\
\uparrow \text { General fatigue } \\
\text { (MFSI-SF) } \\
\text { ( } p<0.05) \\
\uparrow \text { Physical fatigue } \\
\text { (MFSI-SF) } \\
\text { (p<0.05) } \\
\uparrow \text { Emotional } \\
\text { fatigue [MFSI-SF] } \\
(p<0.05)\end{array}$ & $\begin{array}{l}\square \text { Mental fatigue } \\
\text { (MFSI-SF) }\end{array}$ \\
\hline $\begin{array}{c}\text { Zimmer et al., } \\
\text { 2017 } \\
{[39]}\end{array}$ & $\begin{array}{l}\text { Metastasized } \\
\text { colorectal } \\
\text { cancer }\end{array}$ & $\begin{array}{l}\text { Ex: } 68.5 \\
\text { C: } 70\end{array}$ & $\begin{array}{c}\text { CTx }+ \\
\text { TT } \\
\text { no } \\
\text { treat } \\
\text { TT }\end{array}$ & $\begin{array}{c}30 \\
(17 / 13)\end{array}$ & $\begin{array}{c}\text { 8-wk sup } \\
\text { exercise, } \\
\text { combining } \\
\text { endurance, } \\
\text { REX, and } \\
\text { balance exercise }\end{array}$ & $\begin{array}{l}2 \mathrm{2x} / \mathrm{wk}, 60 \mathrm{~min} \\
\text { (Warmup } 10 \mathrm{~min} \text {. } \\
\text { balance training, } \\
\text { AEX } 60-70 \% \text { of } \\
\text { HRmax, } 20 \mathrm{~min}, \\
\text { REX } 5 \text { stations } \\
\text { circuit } 60-80 \% \text { of } \\
\text { h1RM, 8-12 reps, } \\
2 \text { sets 60-80\%\%; } \\
\text { (cool down) }\end{array}$ & Usual care & n.a. * & $\begin{array}{c}\text { EX: } 2 / 17 \\
(11.7 \%) \\
\text { C: } 4 / 13 \\
(30.7 \%) \\
\text { in total } 6 / 30 \\
(20 \%)\end{array}$ & $\begin{array}{c}\text { Mean } \\
\text { training } \\
\text { frequency: } \\
88.3 \%\end{array}$ & 0 & $\begin{array}{c}8 \text { wk: } \\
\uparrow \text { Trial Outcome } \\
\text { Index }(p=0.028) \\
\uparrow \text { Muscle strength } \\
\text { (bench and leg } \\
\text { press, lat } \\
\text { pulldown) } \\
(p=0.002) \\
\uparrow \text { GGT_Reha static } \\
2(p<0.025) \\
14 \text { wk Follow-Up: } \\
\uparrow \text { Trial Outcome } \\
\text { Index ( } p=0.031) \\
\uparrow \text { Muscle strength } \\
\text { (bench and leg } \\
\text { press, lat } \\
\text { pulldown) } \\
(p<0.05)\end{array}$ & $\begin{array}{c}8 \text { wk/14 wk: } \\
\boxminus \text { Physical } \\
\text { Well-being } \\
\text { (FACT/GOG- } \\
\text { NTX) } \\
\square \text { Functional } \\
\text { Well-being } \\
\text { (FACT/GOG- } \\
\text { NTX) } \\
\boxminus \text { Social } \\
\text { Well-being } \\
\text { (FACT/GOG- } \\
\text { NTX) } \\
\square \text { Emotional } \\
\text { Well-being } \\
\text { (FACT/GOG- } \\
\text { NTX) } \\
\square \text { QoL (FACT-G) }\end{array}$ \\
\hline
\end{tabular}

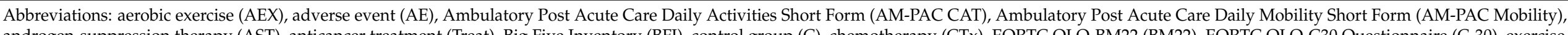

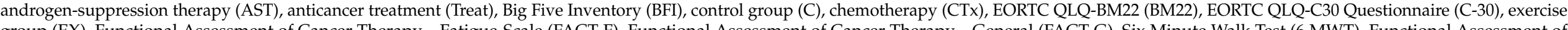

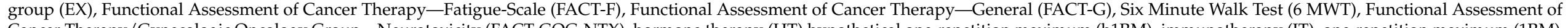

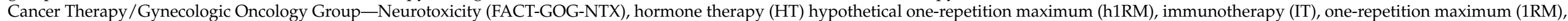

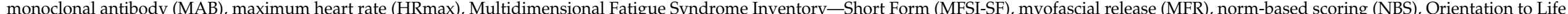

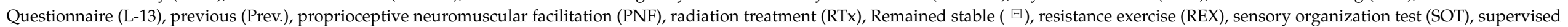

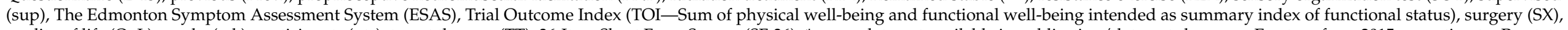

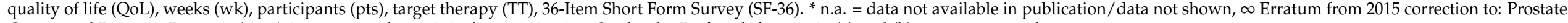
Cancer and Prostatic Diseases (2013) 16, 328-335; doi:10.1038/pcan.2013.22. + Studies by Rief et al. from 2014 (a) and (b) are summarized. 
Table 2. Study characteristics of controlled trials

\begin{tabular}{|c|c|c|c|c|c|c|c|c|c|c|c|c|c|}
\hline Author & Cancer Entities & Age & Treat & $\begin{array}{c}n \\
\text { (EX/C) }\end{array}$ & $\begin{array}{l}\text { Description of } \\
\text { Intervention }\end{array}$ & $\begin{array}{c}\text { Exercise } \\
\text { Modalities }\end{array}$ & Control & Recruitment & $\begin{array}{l}\text { Drop-Out } \\
\text { Rate: }\end{array}$ & Adherence & $\mathrm{AE}$ & $\begin{array}{c}\text { EX vs. UC } \\
\uparrow=\text { Improvement; } \\
\downarrow=\text { Worsening; }\end{array}$ & $\begin{array}{c}\text { EX vs. UC } \\
\text { (Non Sign.) }\end{array}$ \\
\hline $\begin{array}{c}\text { Buss et al., } \\
2009 \\
{[44]}\end{array}$ & $\begin{array}{l}\text { Advanced } \\
\text { cancer patients; } \\
\text { short lifetime } \\
\text { expectancy } \\
\text { (approximately } \\
\text { 1-2 months) }\end{array}$ & n.a. ${ }^{*}$ & none & $\begin{array}{c}57 \\
(38 / 19)\end{array}$ & $\begin{array}{l}\text { 4-wk sup, } \\
\text { individualized } \\
\text { kinesiotherapy }\end{array}$ & $3 x / w k, 20-30 \mathrm{~min}$ & n.a.* & $\begin{array}{l}57 / 80 \\
(71.2 \%)\end{array}$ & $\begin{array}{c}\text { EX: } 12 / 38 \\
(31.6 \%) \\
\text { C: } 2 / 19 \\
(10.5 \%) \\
\text { in total } \\
14 / 57 \\
(24.5 \%) \\
\end{array}$ & n.a. * & n.a. ${ }^{*}$ & $\begin{array}{c}\uparrow \text { Fatigue (BFI) wk } \\
3 \text { and wk } 4 \\
(p<0.001) \\
\downarrow \text { Intensification } \\
\text { physical } \\
\text { symptoms (RSCL) } \\
(p<0.05)\end{array}$ & $\begin{array}{c}\square \text { QoL } \\
\square \text { Mental } \\
\text { symptoms }\end{array}$ \\
\hline $\begin{array}{c}\text { Zhao et al., } \\
2015 \\
{[45]}\end{array}$ & $\begin{array}{c}\text { Head and Neck } \\
\text { squamous cell } \\
\text { cancer, Stage III } \\
\text { and IV } \\
(n=14 \\
\text { oropharynx, } \\
n=1 \text { larynx, } \\
n=1 \\
\text { nasopharynx, } \\
n=2 \text { unknown) }\end{array}$ & $\begin{array}{l}\text { Ex: } 57 \pm 7 \\
\text { C: } 57 \pm 7\end{array}$ & CTx & $\begin{array}{c}20 \\
(11 / 9)\end{array}$ & $\begin{array}{l}\text { 14-wk REX and } \\
\text { walking } \\
\text { exercise (1st } \\
7 \mathrm{wk} \text { sup, } 2 \text { nd } \\
7 \mathrm{wk} \\
\text { home-based) }\end{array}$ & $\begin{array}{c}\text { 3x/wk, } 60 \mathrm{~min}, \\
\text { moderate } \\
\text { intensity, } \\
\text { REX: } 8-12 \text { reps, } \\
3 \text { sets } \\
\text { lking EX: } 30 \mathrm{~min} \\
\text { per wk, } \\
\text { Home-based } \\
\text { exercise } \\
\text { individualized } \\
\text { but also combined } \\
\text { REX and walking } \\
\text { exercise }\end{array}$ & Usual care & $\begin{array}{l}\text { 20/27: } \\
(74.1 \%)\end{array}$ & $\begin{array}{c}\text { EX: } 1 / 11 \\
(9.0 \%) \\
\text { C:2/9 } \\
(22.2 \%) \\
\text { in total } \\
3 / 20 \\
(15 \%)\end{array}$ & $\begin{array}{c}\text { mean session: } \\
15.2 / 21 ; \\
\text { attendance } \\
\text { rate: } 72 \% \\
\text { home- based } \\
\text { training } \\
\text { adherence } \\
\text { n.a. }\end{array}$ & 0 & $\begin{array}{c}7 \text { wk: } \\
\uparrow \text { Vitality } / \text { Fatigue } \\
\text { (SF36) }(p<0.05) \\
\uparrow \text { Mental } \\
\text { Well-being (SF36) } \\
(p<0.05) \\
14 \text { wk: } \\
\uparrow \text { strength knee } \\
\text { extension } \\
(p<0.05) \\
\uparrow \text { Mental } \\
\text { Well-being (SF36) } \\
(p<0.05)\end{array}$ & $\begin{array}{c}7 \mathrm{wk}: \\
\square 6 \mathrm{MWT} \\
\square \text { BMI } \\
\square \text { QoL [SF36] } \\
\square \text { Physical activity } \\
\square \text { Lean body mass } \\
14 \text { wk: } \\
\square 6 \mathrm{MWT} \\
\square \text { BMI } \\
\square \text { QoL [SF36] } \\
\square \text { Physical activity } \\
\square \text { Vitality/Fatigue } \\
{[\text { [SF36) }} \\
\square \text { Lean body mass }\end{array}$ \\
\hline
\end{tabular}

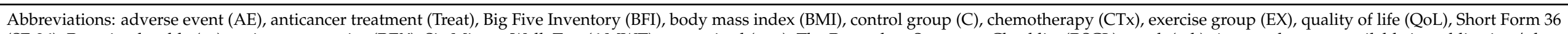

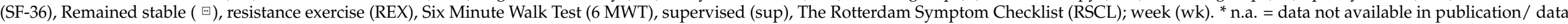
not shown 
Adherence to exercise interventions was defined differently in studies. Five studies did not report adherence to exercise intervention [34,37,40,41,44], while eight studies did report adherence of exercise interventions with a median of 86\% (range: 72-89\%) [18-20,22, 23,25,26,29,30,32]. Adherence changes between trial setting (e.g., supervised/unsupervised or home-based interventions) were not observed.

Overall, ten studies [31,32,35,36,38-40,42,45] reported adverse events as an outcome of measurement, while four studies $[33,34,41,43,44]$ did not assess and report adverse events. In total, $n=940$ patients were observed in the prescribed studies, with $n=220(23.4 \%)$ drop-outs, $n=128$ (58.1\%) patients in the intervention groups, and $n=92(41.8 \%)$ patients in control groups. The primary reason for dropping out was progression of cancer disease or health deterioration $(n=113)$, followed by death due to cancer $(n=61)$. All reasons for drop-outs are displayed in Table 3.

Table 3. Drop-outs with reasons.

\begin{tabular}{cccc}
\hline Drop-Out & In Total & Exercise & Control \\
& $n$ & $n$ & $n$ \\
\hline Participants & 220 & 128 & 92 \\
& $(100 \%)$ & $(58.7 \%)$ & $(41.8 \%)$ \\
\hline Died due to cancer & 61 & 39 & 24 \\
& $(27.7 \%)$ & $(30.5 \%)$ & $(26.0 \%)$ \\
\hline Disease progression/health & 113 & 68 & 45 \\
deterioration & $(51.3 \%)$ & $(53.1 \%)$ & $(48.9 \%)$ \\
\hline Lost to follow-up/ & 35 & 17 & 19.5 \\
non-compliant & $(15.9 \%)$ & $(13.3 \%)$ & $(20 \%)$ \\
\hline Various reasons * & 6 & 1 & 5 \\
& $(2.7 \%)$ & $(0.8 \%)$ & $(5.4 \%)$ \\
\hline Adverse Events *1 & 3 & 3 & 0 \\
& $(1.3 \%)$ & $(2.3 \%)$ & $(0 \%)$ \\
\hline
\end{tabular}

Ex = exercise group; $\mathrm{C}=$ control group; ${ }^{*}$ various reasons can be lack of time, too far to travel, or appointment burdens, etc. ${ }^{* 1}$ In total, 9 adverse events occurred, but only 3 led to drop-out.

Two studies $[36,42]$ reported adverse events, such as NMES-related muscle discomfort Grade 1 after CTC $(n=3)$ [42], muscle pain overuse $(n=4)$, cardiac complaints $(n=1)$, and increased fatigue $(n=1)$ [36]. Only three adverse events resulted in drop-outs, and all occurred in one study [33]. Of patients who received exercise therapy, $n=493$ participants had nine exercise-related adverse events, considered at $2.01 \%$ of all patients who participated in exercise interventions. No further serious adverse events related to exercise were reported.

\subsection{Secondary Outcomes}

\subsubsection{Quality of Life}

Overall QoL was assessed in ten studies [31,34-36,39,40,42-45], with a combined total of 470 patients included. Only two studies $[39,43]$ revealed significant improvement of QoL, with a total sample size of 90 patients $(E X=47, C=43)$. Five studies $[34,40,42,44,45]$ with a combined total sample size of 214 patients $(E X=125, C=89)$ demonstrated a decreased QoL in control group after intervention period while exercise group remained unchanged or slightly increased but without significant statistical values. In three studies $[31,35,36]$, with a total of 166 patients included $(\mathrm{EX}=77, \mathrm{C}=89)$, QoL remained stable/slightly increased in exercise and control groups without significant $p$-values.

\subsubsection{Fatigue}

In total, thirteen studies [31-38,40-42,44,45] with a combined total sample size of 910 patients investigated the influence of exercise on fatigue. Improved fatigue was demonstrated in six studies $[31,33,37,41,44,45]$, with a total of 377 patients observed (EX $=199$, 
$C=178$ ). No statistically relevant changes between groups were detected in six studies $[23,25-27,29,31]$, with a total of 484 patients observed $(E X=247, C=237)$. A worsening of fatigue was shown in one [42] trial with 49 participants $(\mathrm{EX}=30, \mathrm{C}=19$ ).

\subsubsection{Physical Function}

Physical function was assessed in nine studies, whereof four studies $[32,35,38,39]$ with a combined total of 338 participants $(E X=176, C=162)$ revealed improvements in strength-related assessments (1RM, handgrip, bench press, or leg press).

One study [33] with 60 patients $(E X=30, C=30$ ) could prove significant improvement below the anaerobic threshold, while four studies $[35,38,39,45]$ with a combined total of 100 patients $(E X=66, C=61)$ displayed no significant changes. Aerobic exercise capacity improvement was detected in one study [35] with 20 patients $(E X=10, C=10)$, while another one study [38] with 57 patients $(E X=28, C=29)$ could not detect improvement.

\subsubsection{Regular Physical Activity Levels}

Three studies $[35,42,45]$ with a combined total of 89 patients $(E X=51, C=38)$ measured regular physical activity levels. Improvements were noted in $n=1$ study [35] with 20 patients $(E X=10, C=10)$, while two studies $[42,45]$ with a combined total sample size of $69(\mathrm{EX}=41, \mathrm{C}=28)$ displayed no increased physical activity levels.

\subsubsection{Body Composition}

Overall, four studies with a combined total of 166 patients $(E X=89, C=77)$ assessed body composition before and after study participation [35,38,42,45]. Cormie et al. [35], with a sample size of 20 patients $(E X=10, C=10)$, revealed significant improvements of lean body mass in the exercise group. No significant changes were displayed in two studies [38,45] with combined total sample size of $77(\mathrm{EX}=39, \mathrm{C}=38)$. One study [33] with 49 patients ( $E X=30, C=19$ ) evaluated deteriorations of body composition (lean body mass) in both groups after the trial period.

\subsubsection{Sleep}

Only one study [31] with 66 patients $(E X=33, C=33)$ assessed sleep, with a significant improvement demonstrated.

\subsection{Effect According to Intervention \\ 3.6.1. Combined Aerobic and Strength Exercise}

In total, nine studies $[31,32,34-39,45]$ with a combined total of 672 patients $(E X=344$, $C=328$, combined endurance and strength training; $n=8[31,32,34,35,37-39,45]$, with a total of 584 patients ( $E X=302, C=282)$, proved a significant improvement in some domains of physical performance (e.g., shuttle walk test, handgrip, six-minute walking test, bench press and leg press) $(87.5 \%)$, and only one study with 66 patients $(E X=33, C=33$ ) found a significant difference in fatigue scores and sleep quality between exercise and usual care [31]. One study [36] with 88 patients $(E X=42, C=46)$ did not see any improvements after combined exercise intervention. No improvements due to combined intervention could be found in quality of life.

\subsubsection{Single Aerobic Exercise}

Only one study [40] with 42 patients $(E X=21, C=21)$ completed only aerobic exercise, and it did not display any significant changes.

\subsubsection{Single Strength Exercise}

Three RCTs [33,41,42] and one CT [44] with a combined total of 226 patients (EX $=128$ $C=98$ ) performed only strength-related exercise, with significant improvements in fatigue, fatigue subscales, and fatigue severity. One Study [33] with 60 patients $(E X=30, C=30)$ also indicated improvements in quality of life due to strength exercise. One study [42] with 
a sample size of $49(E X=30, C=19)$ displayed no significant changes of fatigue, quality of life, or physical performance compared to usual care.

\subsubsection{Home-Based Exercise}

One study [31] with 66 patients $(E X=33, C=33)$ carried out a full home-based intervention, with significant changes in fatigue, sleep, and mobility. Two studies $[33,45]$ with a combined sample size of 80 patients $(E X=41, C=39)$ performed a mixed trial setting, starting with supervised training and followed by a home-based period, with significant changes related to $30 \mathrm{~s}$ sit-to-stand, fatigue, pain, quality of life, vitality, and strength.

\section{Discussion}

This systematic review summarized the evidence on the feasibility of exercise interventions in ACPs. The results of the presented studies show that exercise interventions are feasible in terms of adverse events, recruitment rate, adherence to intervention, and drop-outs. Reported adverse events appear low grade and reflect common body reactions that might potentially be controlled by an improved training management. However, adverse events have to be considered with caution because grading of adverse events has not been provided. This is in accordance to other published studies related to adverse events while exercising with ACPs [46,47]. Future studies should aim for a detailed collection of adverse events with grading using common terminology criteria for adverse events (CTCAE v5.0 [48]).

Drop-out rates in trials with ACPs show a marked difference compared to trials with tumor patients in earlier stages. The drop-out rate in exercise interventions in a curative treatment setting was reported to be 10-13\% [49,50]. Our aggregated data displayed dropout rates of around $22 \%$. In other supportive and palliative care trials, the drop-out-rates were $26 \%$ with similar reasons to those mentioned in the result section [51], i.e., expected drop-out because of disease progression and health deterioration. This seems to be valuable information when planning new research studies, e.g., for sample size calculations. To clearly identify the influence on the primary endpoint and avoid contamination, around $25 \%$ more patients should be included.

Adherence to exercise interventions was reported in nine of the included studies [31-33,35,36,38,39,42,45], while five studies [34,37,40,41,44] did not report adherence to intervention. The range of adherence was between $72 \%$ and $89 \%$; however, the actual rate could have differed if adherence had been provided in all studies.

Median recruitment rates of exercise studies in the oncology field are $38 \%$ for colorectal cancer patients [52] and 59\% for lung cancer patients in mixed stages I-IV [53]. The median enrollment rate of included studies of our review was $65.7 \%$. Only two studies [32,36] screened more than 200 patients. Studies with a very specific study protocol, for example, studies of Cormie et al. [35] or Rief et al. [33,43], increased the median by screening only a small patient population. Overall, two studies [32,36] screened a high number of patients and pointed out recruitment rates of $68.3 \%$. However, the recruitment process still shows barriers for advanced patients to participate in trials with exercise interventions. It is already well known that staying physically active during cancer treatment is a common challenge and sedentary behavior is present in a high proportion of patients [54]. There are several factors inhibiting patients from participating in exercise activities, even if patients consider exercise to be helpful for physical or psychosocial aspects [55,56]. Barriers to exercise are symptoms and treatment side effects, medical complications, comorbidities, confusion about the capability of exercise, and distance to study sites [57-59]. Physicians and supportive care teams need to identify these individual barriers and find solutions to overcome these to reduce nonparticipation.

However, these studies provide evidence that exercise can be beneficial for improvements or for stabilization of fatigue. Considering the sample size, trials with more than 50 participants $[31,33,37,41]$ showed a significant reduction in fatigue. However, all of the studies raise concerns regarding the overall quality of assessment. A smaller study, 
e.g., the study of Zhao et al. [45], could also show significant improvement of fatigue in combination with overall low risk of bias. Other trials were not able to statistically demonstrate significant differences due to the small sample size. In contrast, the study by Oldervoll et al. [32] with a large study group of 231 patients showed no significant difference in fatigue. In view of the methodological evaluation carried out, the study cited reveals four domains of the RoB 2.0 as of concern. The authors also discuss reasons for not generating significant results as progression of the disease within the intervention period and a significantly increased baseline fatigue compared to other trials. Cancer-related fatigue affects almost all ACPs and correlates with the quality of life; high fatigue indicates a lower quality of life [60]. Furthermore, increased fatigue levels lead to physical inactivity and consequently, loss of physical performance and muscle mass [61]. The included studies conducted different interventional modalities (e.g., home-based, supervised, resistance exercise, combined resistance endurance exercise, Tai-Chi, intensity, duration, frequency) that influenced fatigue positively. Cancer-related fatigue is known to alter different biological pathways during the trajectory of cancer, such as muscle metabolism or pro-inflammatory cytokines, due to the tumor itself or as a treatment side effect [62,63]. Exercise can reverse this biological process and alleviate the expression of different biological pathways [64]. Further research should address which exercise principles are most beneficial. It remains unclear up to which point interventions against fatigue should be applied; the European Association for Palliative Care (EAPC) discusses that in the end-of-life stage, fatigue can also give a protection or shield [65]. Interventional treatment for fatigue could have a harmful on this protection and affect quality of life.

Considering the methodological quality of the provided studies and the sample size, it appears that exercise with ACPs can improve or at least stabilize QoL. The studies we rated with an overall low risk of bias [36,39] both showed an increase in QoL, although this difference was not significant in the study of Poort et al. [36]. Poort et al. had already mentioned the high drop-out in exercise group as a potential reason. Overall, the sample sizes of studies with QoL as an endpoint are mostly small, and evidence is limited regarding QoL as an endpoint.

Several studies underlined the positive effects of exercise on physical performance and function in ACPs [31-35,38,39,45]. Especially, studies with more than 50 participants [3133,38 ] showed an improvement in physical domains. Furthermore, studies rated with a low overall risk of bias $[38,39]$ also showed significant improvement despite the small sample size. Exercise in ACPs can significantly improve physical performance. Loss of physical function significantly correlates with low quality of life in ACPs [66]. Decline of physical function is a predictor for negative changes in mental components of health-related quality of life [67]. Therefore, exercise could be a considered a promising solution to improve physical function and improve associated patients' related outcomes (PROMS). The intervention context was mostly supervised with combined strength and endurance units [32-35,38,45]. Trial settings with home-based parts seem to be promising as well and revealed positive results on PROMS. Three studies [31,32,43,45] solely included home-based interventions or mixed models with supervised sessions followed by home-based training. Home-based training seems to be impactful as well, and could be a future option to lower barriers to improve PROs. Multidimensional cancer rehabilitation delivered online in early cancer and survivorship had a high acceptability and also revealed significant improvements in physical function [68]. Macdonald et al., 2020, displayed a solid recruitment rate (64\%) and impressive retention rate $(83 \%)$ and adherence rates $(80 \%)$. Future research should address the question of supervised vs. home-based training in ACPs. Over the last decades, the quantity of telemedicine-delivered interventions has increased rapidly, providing a promising opportunity for advanced cancer exercise trials. Home-based interventions delivered by telemedicine are proven to be feasible and safe $[69,70]$. The application of telemedicine could significantly reduce barriers of ACPs and reduce additional burdens of participation. 


\section{Limitations}

The main weakness of the review is the high heterogeneity of the different studies. Meta-analysis could not be performed due to the different test procedures or questionnaires. Overall, studies have a small sample size, and some studies were unclear about the comparison to exercise and intensity of exercise that was completed by exercise participants. Risk of bias indicates some methodical variations in nearly all provided studies. Most of the described studies have some methodical concerns. Moreover, we did not search all possible literature sources for exercise studies in ACPs because of linguistic limitations. As a result, studies may be missing. However, exercise seems feasible, potent to increase physical performance, and may lower disease-specific symptoms, such as fatigue. Exercise is cost efficient and easy to apply as a supportive management tool. Patients who are working out actively have the opportunity to actively improve their living situation. This strengthens resilience against the life-shortening illnesses.

\section{Conclusions}

Regardless of the heterogeneous study situation within the evaluated trials, exercise interventions seem to be safe and feasible in ACPs. The summarized evidence indicates beneficial effects of exercise on physical performance and QoL, and a potential reduction of fatigue. The combination of aerobic and strength exercise seems preferable to improve physical function. Further research with complete reporting of the exercise adherence should examine these preliminary beneficial effects in multicenter studies to provide evidence regarding the ability of different exercise modalities to lower symptom burden and improve PROs. Based on our results, exercise should be offered to ACPs as well as people receiving curative treatment.

Author Contributions: Conceptualization, N.D.L., M.G.; methodology, N.D.L., T.N. and M.G.; formal analysis, N.D.L. and T.N.; investigation, N.D.L., T.N. and M.G.; resources, palliative care and medical input, M.T.; writing—original draft preparation, N.D.L.; writing-review and editing, T.N., M.G. and M.T.; visualization, N.D.L., T.N. and M.G.; supervision, M.T. and M.G.; project administration, N.D.L., T.N. and M.G. All authors have read and agreed to the published version of the manuscript.

Funding: This research received no external funding.

Institutional Review Board Statement: Not applicable.

Informed Consent Statement: Not applicable.

Data Availability Statement: Publicly available datasets were analyzed in this study. This data can be found in the cited publications from reference [31-45].

Conflicts of Interest: The authors declare no conflict of interest.

\section{References}

1. WHO. Data and Statistics on Cancer in Europe. Available online: https://www.euro.who.int/en/health-topics/noncommunicablediseases/cancer/data-and-statistics (accessed on 28 January 2021).

2. Pinto, B.M.; Trunzo, J.J. Health behaviors during and after a cancer diagnosis. Cancer 2005, 104, 2614-2623. [CrossRef]

3. Temel, J.S.; Greer, J.A.; Muzikansky, A.; Gallagher, E.R.; Admane, S.; Jackson, V.A.; Dahlin, C.M.; Blinderman, C.D.; Jacobsen, J.; Pirl, W.F. Early palliative care for patients with metastatic non-small-cell lung cancer. N. Engl. J. Med. 2010, 363, 733-742. [CrossRef] [PubMed]

4. $\quad$ Basch, E.; Deal, A.M.; Dueck, A.C.; Scher, H.I.; Kris, M.G.; Hudis, C.; Schrag, D. Overall Survival Results of a Trial Assessing Patient-Reported Outcomes for Symptom Monitoring During Routine Cancer Treatment. JAMA 2017, 318, 197-198. [CrossRef]

5. Potter, J.; Hami, F.; Bryan, T.; Quigley, C. Symptoms in 400 patients referred to palliative care services: Prevalence and patterns. Palliat. Med. 2003, 17, 310-314. [CrossRef]

6. Tewes, M.; Baumann, F.; Teufel, M.; Ostgathe, C. Symptoms During Outpatient Cancer Treatment and Options for Their Management. Dtsch. Arztebl. Int. 2021, 118. [CrossRef]

7. Al Maqbali, M.; Al Sinani, M.; Al Naamani, Z.; Al Badi, K. Prevalence of Fatigue in Patients with Cancer: A Systematic Review and Meta-Analysis. J. Pain Symptom Manag. 2020, 61, 167-189. [CrossRef] [PubMed] 
8. Van Lancker, A.; Velghe, A.; Van Hecke, A.; Verbrugghe, M.; Van Den Noortgate, N.; Grypdonck, M.; Verhaeghe, S.; Bekkering, G.; Beeckman, D. Prevalence of symptoms in older cancer patients receiving palliative care: A systematic review and meta-analysis. J. Pain Symptom Manag. 2014, 47, 90-104. [CrossRef]

9. Rha, S.Y.; Lee, J. Symptom clusters during palliative chemotherapy and their influence on functioning and quality of life. Support. Care Cancer 2017, 25, 1519-1527. [CrossRef] [PubMed]

10. Christensen, J.F.; Simonsen, C.; Hojman, P. Exercise training in cancer control and treatment. Compr. Physiol. $2011,9,165-205$.

11. Patel, A.V.; Friedenreich, C.M.; Moore, S.C.; Hayes, S.C.; Silver, J.K.; Campbell, K.L.; Winters-Stone, K.; Gerber, L.H.; George, S.M.; Fulton, J.E. American College of Sports Medicine roundtable report on physical activity, sedentary behavior, and cancer prevention and control. Med. Sci. Sports Exerc. 2019, 51, 2391. [CrossRef] [PubMed]

12. Mustian, K.M.; Alfano, C.M.; Heckler, C.; Kleckner, A.S.; Kleckner, I.R.; Leach, C.R.; Mohr, D.; Palesh, O.G.; Peppone, L.J.; Piper, B.F. Comparison of pharmaceutical, psychological, and exercise treatments for cancer-related fatigue: A meta-analysis. JAMA Oncol. 2017, 3, 961-968. [CrossRef] [PubMed]

13. Courneya, K.S.; McKenzie, D.C.; Mackey, J.R.; Gelmon, K.; Friedenreich, C.M.; Yasui, Y.; Reid, R.D.; Cook, D.; Jespersen, D.; Proulx, C. Effects of exercise dose and type during breast cancer chemotherapy: Multicenter randomized trial. J. Natl. Cancer Inst. 2013, 105, 1821-1832. [CrossRef]

14. Brown, J.C.; Damjanov, N.; Courneya, K.S.; Troxel, A.B.; Zemel, B.S.; Rickels, M.R.; Ky, B.; Rhim, A.D.; Rustgi, A.K.; Schmitz, K.H. A randomized dose-response trial of aerobic exercise and health-related quality of life in colon cancer survivors. Psychooncology 2018, 27, 1221-1228. [CrossRef]

15. Nakano, J.; Hashizume, K.; Fukushima, T.; Ueno, K.; Matsuura, E.; Ikio, Y.; Ishii, S.; Morishita, S.; Tanaka, K.; Kusuba, Y. Effects of Aerobic and Resistance Exercises on Physical Symptoms in Cancer Patients: A Meta-analysis. Integr. Cancer Ther. 2018, 17, 1048-1058. [CrossRef]

16. van Waart, H.; Stuiver, M.M.; van Harten, W.H.; Geleijn, E.; Kieffer, J.M.; Buffart, L.M.; de Maaker-Berkhof, M.; Boven, E.; Schrama, J.; Geenen, M.M.; et al. Effect of Low-Intensity Physical Activity and Moderate- to High-Intensity Physical Exercise During Adjuvant Chemotherapy on Physical Fitness, Fatigue, and Chemotherapy Completion Rates: Results of the PACES Randomized Clinical Trial. J. Clin. Oncol. 2015, 33, 1918-1927. [CrossRef]

17. Tian, L.; Lu, H.J.; Lin, L.; Hu, Y. Effects of aerobic exercise on cancer-related fatigue: A meta-analysis of randomized controlled trials. Support. Care Cancer 2016, 24, 969-983. [CrossRef]

18. Scott, J.M.; Zabor, E.C.; Schwitzer, E.; Koelwyn, G.J.; Adams, S.C.; Nilsen, T.S.; Moskowitz, C.S.; Matsoukas, K.; Iyengar, N.M.; Dang, C.T.; et al. Efficacy of Exercise Therapy on Cardiorespiratory Fitness in Patients With Cancer: A Systematic Review and Meta-Analysis. J. Clin. Oncol. 2018, 36, 2297-2305. [CrossRef] [PubMed]

19. Strasser, B.; Steindorf, K.; Wiskemann, J.; Ulrich, C.M. Impact of resistance training in cancer survivors: A meta-analysis. Med. Sci. Sports Exerc. 2013, 45, 2080-2090. [CrossRef]

20. Winters-Stone, K.; Dobek, J.; Nail, L.; Bennett, J.; Leo, M.; Torgrimson-Ojerio, B.; Luoh, S.-W.; Schwartz, A. Impact+ resistance training improves bone health and body composition in prematurely menopausal breast cancer survivors: A randomized controlled trial. Osteoporos. Int. 2013, 24, 1637-1646. [CrossRef] [PubMed]

21. Courneya, K.S.; Segal, R.J.; Mackey, J.R.; Gelmon, K.; Reid, R.D.; Friedenreich, C.M.; Ladha, A.B.; Proulx, C.; Vallance, J.K.; Lane, K.; et al. Effects of aerobic and resistance exercise in breast cancer patients receiving adjuvant chemotherapy: A multicenter randomized controlled trial. J. Clin. Oncol. 2007, 25, 4396-4404. [CrossRef]

22. Zhu, G.; Zhang, X.; Wang, Y.; Xiong, H.; Zhao, Y.; Sun, F. Effects of exercise intervention in breast cancer survivors: A meta-analysis of 33 randomized controlled trails. Onco Targets Ther. 2016, 9, 2153-2168. [CrossRef]

23. Mustian, K.M.; Griggs, J.J.; Morrow, G.R.; McTiernan, A.; Roscoe, J.A.; Bole, C.W.; Atkins, J.N.; Issell, B.F. Exercise and side effects among 749 patients during and after treatment for cancer: A University of Rochester Cancer Center Community Clinical Oncology Program Study. Support. Care Cancer 2006, 14, 732-741. [CrossRef]

24. Andersen, C.; Adamsen, L.; Moeller, T.; Midtgaard, J.; Quist, M.; Tveteraas, A.; Rorth, M. The effect of a multidimensional exercise programme on symptoms and side-effects in cancer patients undergoing chemotherapy-the use of semi-structured diaries. Eur. J. Oncol. Nurs. 2006, 10, 247-262. [CrossRef]

25. Piraux, E.; Caty, G.; Aboubakar Nana, F.; Reychler, G. Effects of exercise therapy in cancer patients undergoing radiotherapy treatment: A narrative review. SAGE Open Med. 2020, 8, 2050312120922657. [CrossRef] [PubMed]

26. Schumann, M.; Freitag, N.; Bloch, W. Advanced Exercise Prescription for Cancer Patients and its Application in Germany. J. Sci. Sport Exerc. 2020, 2, 201-214. [CrossRef]

27. Heywood, R.; McCarthy, A.L.; Skinner, T.L. Efficacy of exercise interventions in patients with advanced cancer: A systematic review. Arch. Phys. Med. Rehabil. 2018, 99, 2595-2620. [CrossRef]

28. Page, M.J.; McKenzie, J.E.; Bossuyt, P.M.; Boutron, I.; Hoffmann, T.C.; Mulrow, C.D.; Shamseer, L.; Tetzlaff, J.M.; Akl, E.A.; Brennan, S.E.; et al. The PRISMA 2020 statement: An updated guideline for reporting systematic reviews. BMJ 2021, 372, n71. [CrossRef]

29. Sterne, J.A.C.; Savovic, J.; Page, M.J.; Elbers, R.G.; Blencowe, N.S.; Boutron, I.; Cates, C.J.; Cheng, H.Y.; Corbett, M.S.; Eldridge, S.M.; et al. RoB 2: A revised tool for assessing risk of bias in randomised trials. BMJ 2019, 366, 14898. [CrossRef] [PubMed] 
30. Sterne, J.A.; Hernan, M.A.; Reeves, B.C.; Savovic, J.; Berkman, N.D.; Viswanathan, M.; Henry, D.; Altman, D.G.; Ansari, M.T.; Boutron, I.; et al. ROBINS-I: A tool for assessing risk of bias in non-randomised studies of interventions. BMJ 2016, 355 , i4919. [CrossRef]

31. Cheville, A.L.; Kollasch, J.; Vandenberg, J.; Shen, T.; Grothey, A.; Gamble, G.; Basford, J.R. A home-based exercise program to improve function, fatigue, and sleep quality in patients with Stage IV lung and colorectal cancer: A randomized controlled trial. $J$. Pain Symptom Manag. 2013, 45, 811-821. [CrossRef] [PubMed]

32. Oldervoll, L.M.; Loge, J.H.; Lydersen, S.; Paltiel, H.; Asp, M.B.; Nygaard, U.V.; Oredalen, E.; Frantzen, T.L.; Lesteberg, I.; Amundsen, L.; et al. Physical exercise for cancer patients with advanced disease: A randomized controlled trial. Oncologist 2011, 16, 1649-1657. [CrossRef] [PubMed]

33. Rief, H.; Omlor, G.; Akbar, M.; Welzel, T.; Bruckner, T.; Rieken, S.; Haefner, M.F.; Schlampp, I.; Gioules, A.; Habermehl, D. Feasibility of isometric spinal muscle training in patients with bone metastases under radiation therapy-first results of a randomized pilot trial. BMC Cancer 2014, 14, 1-10. [CrossRef]

34. Henke, C.C.; Cabri, J.; Fricke, L.; Pankow, W.; Kandilakis, G.; Feyer, P.C.; de Wit, M. Strength and endurance training in the treatment of lung cancer patients in stages IIIA/IIIB/IV. Support. Care Cancer 2014, 22, 95-101. [CrossRef]

35. Cormie, P.; Newton, R.U.; Spry, N.; Joseph, D.; Taaffe, D.R.; Galvao, D.A. Safety and efficacy of resistance exercise in prostate cancer patients with bone metastases. Prostate Cancer Prostatic Dis. 2013, 16, 328-335. [CrossRef]

36. Poort, H.; Peters, M.; van der Graaf, W.T.A.; Nieuwkerk, P.T.; van de Wouw, A.J.; Nijhuis-van der Sanden, M.W.G.; Bleijenberg, G.; Verhagen, C.; Knoop, H. Cognitive behavioral therapy or graded exercise therapy compared with usual care for severe fatigue in patients with advanced cancer during treatment: A randomized controlled trial. Ann. Oncol. 2020, 31, 115-122. [CrossRef]

37. Zhou, W.; Wan, Y.H.; Chen, Q.; Qiu, Y.R.; Luo, X.M. Effects of Tai Chi Exercise on Cancer-Related Fatigue in Patients with Nasopharyngeal Carcinoma Undergoing Chemoradiotherapy: A Randomized Controlled Trial. J. Pain Symptom Manag. 2018, 55, 737-744. [CrossRef]

38. Galvao, D.A.; Taaffe, D.R.; Spry, N.; Cormie, P.; Joseph, D.; Chambers, S.K.; Chee, R.; Peddle-McIntyre, C.J.; Hart, N.H.; Baumann, F.T.; et al. Exercise Preserves Physical Function in Prostate Cancer Patients with Bone Metastases. Med. Sci. Sports Exerc. 2018, 50, 393-399. [CrossRef]

39. Zimmer, P.; Trebing, S.; Timmers-Trebing, U.; Schenk, A.; Paust, R.; Bloch, W.; Rudolph, R.; Streckmann, F.; Baumann, F.T. Eight-week, multimodal exercise counteracts a progress of chemotherapy-induced peripheral neuropathy and improves balance and strength in metastasized colorectal cancer patients: A randomized controlled trial. Support. Care Cancer 2018, 26, 615-624. [CrossRef] [PubMed]

40. Tsianakas, V.; Harris, J.; Ream, E.; Van Hemelrijck, M.; Purushotham, A.; Mucci, L.; Green, J.S.; Fewster, J.; Armes, J. CanWalk: A feasibility study with embedded randomised controlled trial pilot of a walking intervention for people with recurrent or metastatic cancer. BMJ Open 2017, 7, e013719. [CrossRef]

41. Pyszora, A.; Budzynski, J.; Wojcik, A.; Prokop, A.; Krajnik, M. Physiotherapy programme reduces fatigue in patients with advanced cancer receiving palliative care: Randomized controlled trial. Support. Care Cancer 2017, 25, 2899-2908. [CrossRef] [PubMed]

42. Maddocks, M.; Halliday, V.; Chauhan, A.; Taylor, V.; Nelson, A.; Sampson, C.; Byrne, A.; Griffiths, G.; Wilcock, A. Neuromuscular electrical stimulation of the quadriceps in patients with non-small cell lung cancer receiving palliative chemotherapy: $\mathrm{A}$ randomized phase II study. PLoS ONE 2013, 8, e86059. [CrossRef]

43. Rief, H.; Akbar, M.; Keller, M.; Omlor, G.; Welzel, T.; Bruckner, T.; Rieken, S.; Häfner, M.F.; Schlampp, I.; Gioules, A. Quality of life and fatigue of patients with spinal bone metastases under combined treatment with resistance training and radiation therapy-a randomized pilot trial. Radiat. Oncol. 2014, 9, 1-8. [CrossRef] [PubMed]

44. Buss, T.; de Walden-Galuszko, K.; Modlinska, A.; Osowicka, M.; Lichodziejewska-Niemierko, M.; Janiszewska, J. Kinesitherapy alleviates fatigue in terminal hospice cancer patients-an experimental, controlled study. Support. Care Cancer 2010, 18, 743-749. [CrossRef]

45. Zhao, S.G.; Alexander, N.B.; Djuric, Z.; Zhou, J.; Tao, Y.; Schipper, M.; Feng, F.Y.; Eisbruch, A.; Worden, F.P.; Strath, S.J.; et al. Maintaining physical activity during head and neck cancer treatment: Results of a pilot controlled trial. Head Neck 2016, 38 (Suppl. 1), E1086-E1096. [CrossRef]

46. Gauß, F.G.; Beller, R.; Boos, J.; Däggelmann, J.; Stalf, H.; Wiskemann, J.; Götte, M. Adverse events during supervised exercise interventions in Pediatric Oncology-a nationwide survey. Front. Pediatrics 2021, 9, 568. [CrossRef]

47. Clauss, D.; Quirmbach, F.; Wiskemann, J.; Rosenberger, F. Adverse Events beim Training mit onkologischen Patienten: Wie sicher ist das Training außerhalb klinischer Studien? B G Beweg. Gesundh. 2019, 35, 194-201. [CrossRef]

48. National Cancer Institute, N.C. Common Terminology Criteria for Adverse Events (CTCAE). Available online: https://ctep. cancer.gov/protocoldevelopment/electronic_applications/docs/CTCAE_v5_Quick_Reference_5x7.pdf (accessed on 10 June 2021).

49. Steins Bisschop, C.N.; Courneya, K.S.; Velthuis, M.J.; Monninkhof, E.M.; Jones, L.W.; Friedenreich, C.; van der Wall, E.; Peeters, P.H.; May, A.M. Control group design, contamination and drop-out in exercise oncology trials: A systematic review. PLoS ONE 2015, 10, e0120996. [CrossRef] 
50. Bullard, T.; Ji, M.; An, R.; Trinh, L.; Mackenzie, M.; Mullen, S.P. A systematic review and meta-analysis of adherence to physical activity interventions among three chronic conditions: Cancer, cardiovascular disease, and diabetes. BMC Public Health 2019, 19, 636. [CrossRef] [PubMed]

51. Hui, D.; Glitza, I.; Chisholm, G.; Yennu, S.; Bruera, E. Attrition rates, reasons, and predictive factors in supportive care and palliative oncology clinical trials. Cancer 2013, 119, 1098-1105. [CrossRef] [PubMed]

52. Singh, B.; Hayes, S.C.; Spence, R.R.; Steele, M.L.; Millet, G.Y.; Gergele, L. Exercise and colorectal cancer: A systematic review and meta-analysis of exercise safety, feasibility and effectiveness. Int. J. Behav. Nutr. Phys. Act. 2020, 17, 122. [CrossRef]

53. Singh, B.; Spence, R.; Steele, M.L.; Hayes, S.; Toohey, K. Exercise for Individuals with Lung Cancer: A Systematic Review and Meta-Analysis of Adverse Events, Feasibility, and Effectiveness. Semin. Oncol. Nurs. 2020, 36, 151076. [CrossRef] [PubMed]

54. Fassier, P.; Zelek, L.; Partula, V.; Srour, B.; Bachmann, P.; Touillaud, M.; Druesne-Pecollo, N.; Galan, P.; Cohen, P.; Hoarau, H.; et al. Variations of physical activity and sedentary behavior between before and after cancer diagnosis: Results from the prospective population-based NutriNet-Sante cohort. Medicine 2016, 95, e4629. [CrossRef]

55. Mikkelsen, M.K.; Nielsen, D.L.; Vinther, A.; Lund, C.M.; Jarden, M. Attitudes towards physical activity and exercise in older patients with advanced cancer during oncological treatment-A qualitative interview study. Eur. J. Oncol. Nurs. 2019, 41, 16-23. [CrossRef]

56. Elshahat, S.; Treanor, C.; Donnelly, M. Factors influencing physical activity participation among people living with or beyond cancer: A systematic scoping review. Int. J. Behav. Nutr. Phys. Act. 2021, 18, 50. [CrossRef]

57. Frikkel, J.; Gotte, M.; Beckmann, M.; Kasper, S.; Hense, J.; Teufel, M.; Schuler, M.; Tewes, M. Fatigue, barriers to physical activity and predictors for motivation to exercise in advanced Cancer patients. BMC Palliat. Care 2020, 19, 43. [CrossRef] [PubMed]

58. Kartolo, A.; Cheng, S.; Petrella, T. Motivation and preferences of exercise programmes in patients with inoperable metastatic lung cancer: A need assessment. Support. Care Cancer 2016, 24, 129-137. [CrossRef]

59. Adamsen, L.; Stage, M.; Laursen, J.; Rorth, M.; Quist, M. Exercise and relaxation intervention for patients with advanced lung cancer: A qualitative feasibility study. Scand. J. Med. Sci. Sports 2012, 22, 804-815. [CrossRef] [PubMed]

60. Charalambous, A.; Kouta, C. Cancer Related Fatigue and Quality of Life in Patients with Advanced Prostate Cancer Undergoing Chemotherapy. Biomed. Res. Int. 2016, 2016, 3989286. [CrossRef] [PubMed]

61. Kummer, F.; Catuogno, S.; Perseus, J.M.; Bloch, W.; Baumann, F.T. Relationship between cancer-related fatigue and physical activity in inpatient cancer rehabilitation. Anticancer. Res. 2013, 33, 3415-3422.

62. Bower, J.E. Cancer-related fatigue-mechanisms, risk factors, and treatments. Nat. Rev. Clin. Oncol. 2014, 11, 597-609. [CrossRef]

63. Barsevick, A.; Frost, M.; Zwinderman, A.; Hall, P.; Halyard, M.; Consortium, G. I'm so tired: Biological and genetic mechanisms of cancer-related fatigue. Qual. Life Res. 2010, 19, 1419-1427. [CrossRef] [PubMed]

64. Hardee, J.P.; Counts, B.R.; Carson, J.A. Understanding the Role of Exercise in Cancer Cachexia Therapy. Am. J. Lifestyle Med. 2019, 13, 46-60. [CrossRef]

65. Radbruch, L.; Strasser, F.; Elsner, F.; Gonçalves, J.F.; Løge, J.; Kaasa, S.; Nauck, F.; Stone, P.; the Research Steering Committee of the European Association for Palliative Care (EAPC). Fatigue in palliative care patients-An EAPC approach. Palliat. Med. 2008, 22, 13-32. [CrossRef] [PubMed]

66. Park, S.-A.; Chung, S.H.; Lee, Y. Factors influencing the quality of life of patients with advanced cancer. Appl. Nurs. Res. 2017, 33, 108-112. [CrossRef] [PubMed]

67. Shallwani, S.M.; Simmonds, M.J.; Kasymjanova, G.; Spahija, J. Quality of life, symptom status and physical performance in patients with advanced non-small cell lung cancer undergoing chemotherapy: An exploratory analysis of secondary data. Lung Cancer 2016, 99, 69-75. [CrossRef] [PubMed]

68. MacDonald, A.M.; Chafranskaia, A.; Lopez, C.J.; Maganti, M.; Bernstein, L.J.; Chang, E.; Langelier, D.M.; Obadia, M.; Edwards, B.; Oh, P.; et al. CaRE @ Home: Pilot Study of an Online Multidimensional Cancer Rehabilitation and Exercise Program for Cancer Survivors. J. Clin. Med. 2020, 9, 92. [CrossRef]

69. Bland, K.A.; Bigaran, A.; Campbell, K.L.; Trevaskis, M.; Zopf, E.M. Exercising in isolation? The role of telehealth in exercise oncology during the COVID-19 pandemic and beyond. Phys. Ther. 2020, 100, 1713-1716. [CrossRef] [PubMed]

70. Morrison, K.S.; Paterson, C.; Toohey, K. The feasibility of exercise interventions delivered via telehealth for people affected by cancer: A rapid review of the literature. Semin. Oncol. Nurs. 2020, 36, 151092. [CrossRef] 